\title{
Driven Lattice Gases with Quenched Disorder: Exact Results and Different Macroscopic Regimes
}

\author{
Goutam Tripathy and Mustansir Barma \\ Theoretical Physics Group, Tata Institute of Fundamental Research, Homi Bhabha Road, Mumbai 400005
}

August 14, 2018

\begin{abstract}
We study the effect of quenched spatial disorder on the steady states of driven systems of interacting particles. Two sorts of models are studied: disordered drop-push processes and their generalizations, and the disordered asymmetric simple exclusion process. We write down the exact steady-state measure, and consequently a number of physical quantities explicitly, for the drop-push dynamics in any dimensions for arbitrary disorder. We find that three qualitatively different regimes of behaviour are possible in 1- $d$ disordered driven systems. In the Vanishing-Current regime, the steady-state current approaches zero in the thermodynamic limit. A system with a non-zero current can either be in the Homogeneous regime, chracterized by a single macroscopic density, or the Segregated-Density regime, with macroscopic regions of different densities. We comment on certain important constraints to be taken care of in any field theory of disordered systems.
\end{abstract}

PACS numbers: 05.60.+w, 47.55.Mh, 64.60.-i, 05.50.+q

\section{INTRODUCTION}

It is known that quenched disorder can strongly affect the large-scale, long-time behaviour of nonequilibrium driven systems with interacting constituents. The interplay of disorder, interactions and drive opens up the possibility of new regimes of complex and interesting behaviour arising in these systems [1]. In the theoretical effort to delineate and explore regimes of new behaviour, an important role is played by simple models which capture some features of more complex physical systems. In this paper, we study disordered driven diffusive systems by analysing stochastically evolving lattice gas models, with quenched disordered hopping rates [2].

Driven diffusive systems in the absence of disorder have been studied extensively and are reviewed in [3]. Also, systems with disorder and drive but no interactions between particles are well studied and understood [1]. But there have been only sporadic studies of disordered driven diffusive systems of interacting particles. It has been argued that strong enough random site dilution can substantially affect the transport properties of particles with hard-core interactions, and can make the system respond nonmonotonically to the driving field [5.6. 6 . On the other hand, a low concentration of blocked sites was found numerically not to affect the critical behaviour of a driven lattice gas with additional attractive inter-particle interactions [7]. Finally, a driven lattice gas with a quenched noise distribution was studied using field-theoretic techniques in [8], but the connection of this study with particle-conserving disordered lattice gas models is not clear.

In this paper, we study disordered lattice gas models with a view towards identifying different sorts of generic behaviour that can arise on large scales as a consequence of disorder. The only interaction included is the hardcore constraint which limits the allowed occupancy of each site. Our results pertain mostly, but not exclusively, to one dimension. In the remainder of this Introduction, we discuss the different types of behaviour displayed by the lattice gas models under study.

We find three distinct regimes in disordered driven diffusive systems in one dimension:

In the Homogeneous regime, the state of the system is characterized by a single density and a nonzero current. Quenched disorder induces variations of the density on the microscopic scale, of the order of a few lattice spacings. However, the system has a macroscopically homogeneous density. In the thermodynamic limit, the current approaches a finite value.

In the Segregated-Density regime, the state of the system is characterized by two distinct values of density, and a nonzero current. Besides microscopic-scale variations of the density, there are macroscopic regions with differing high and low densities. The state is thus characterized by phase separation of the density, and a spatially constant time-averaged current which remains finite in the thermodynamic limit.

In the Vanishing-Current regime, the state of the system is characterized by two distinct values of the density, and an essentially zero current. The hallmark of this regime is that the current decreases as the system size increases, and vanishes in the thermodynamic limit. This is a consequence of rare but rate-limiting backbends, or stretches of bonds which disfavour the forward flow of current. The density is inhomogeneous on a macroscopic scale.

The density profiles in typical states in each of the three regimes are depicted in Figure 1, while Figure 2 shows the variation of the current with system size in the three cases. 

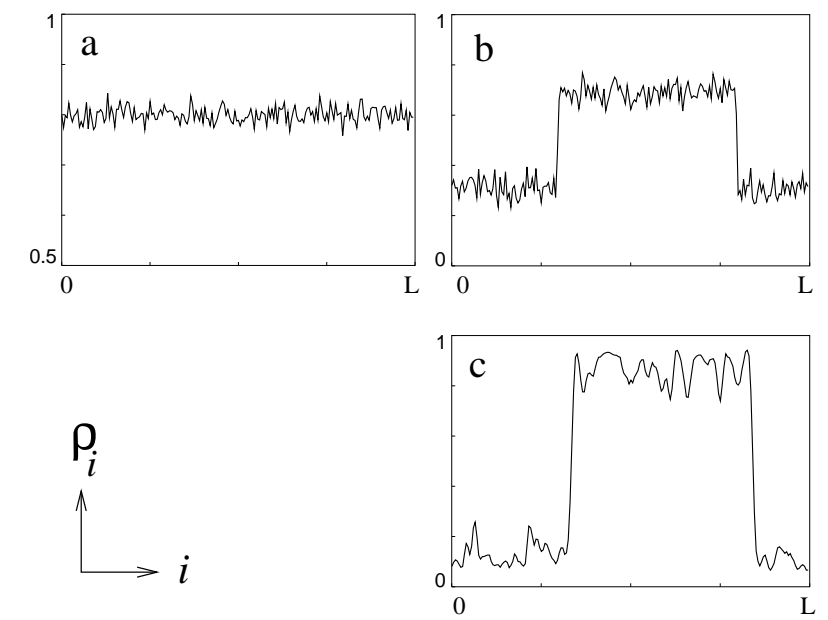

FIG. 1. Representative steady-state density profiles for the (a) Homogeneous (b) Segregated-Density and (c) Vanishing-Current regimes in the Disordered Asymmetric Exclusion Process (DASEP).

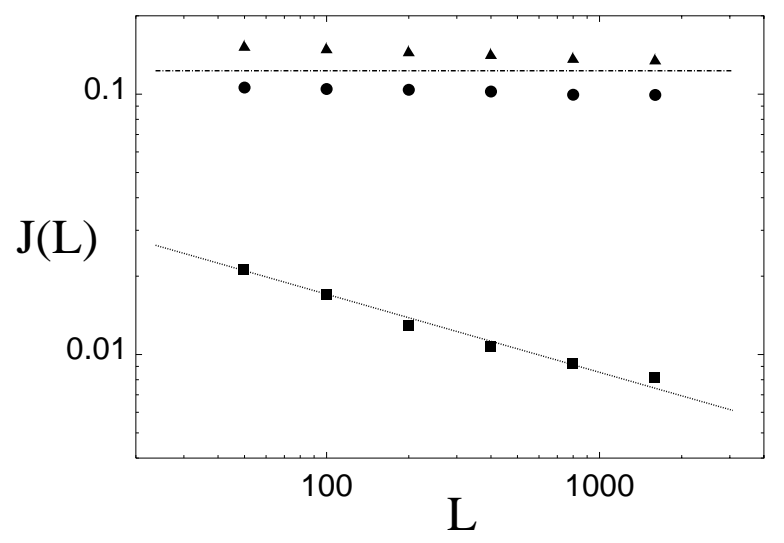

FIG. 2. Variation of the steady-state current with the system size for the three DASEP regimes of Fig. 1: (a) Homogeneous (circles), (b) Segregated-Density (triangles) and (c) Vanishing-Current (squares). In (a) and (b), the current approaches a finite value in the thermodynamic limit whereas in (c) the current vanishes as a power of the system size. The dashed line corresponds to $J=0.125$ which is the limiting value of the current in the regime (b) for the chosen values of the parameters.

Examples of these behaviours are discussed in this paper for two types of lattice-gas models, namely the disordered drop-push process (DDPP) and the disordered asymmetric simple exclusion process (DASEP). The models are defined in detail in Sections II and III respectively, but for the purpose of discussion here, it suffices to note that the models are similar in that there is a maximum occupancy of each site in both, and are different in the dynamical moves - attempted nearest neighbour jumps in the DASEP, and slightly longer-ranged leapfrogging moves in the DDPP.
The absence of detailed balance, together with the breaking of translational invariance, in disordered offequilibrium systems makes the characterization of even the stationary state difficult in general. It is shown that the steady state of the disordered drop-push process can be found explicitly - the first such instance we are aware of, in a system with disorder, interactions and drive [9]. This determination - which is based on the condition of pairwise balance [10] - shows that a product measure form is valid in all dimensions. The form reflects the microscopic inhomogeneities coming from the underlying disorder, and results in a macroscopically homogeneous state.

For the disordered asymmetric exclusion process, the steady state measure is not analytically characterizable, and we study the problem within a site-wise inhomogeneous mean-field theory and by numerical simulation. The result depends crucially on whether or not the system has backbends, which are stretches of the lattice where the local bias is against the particle flow. In the no-backbend case, when the average particle density is sufficiently away from $1 / 2$, the spatial profile of the density has microscopic shocks, but is uniform on macroscopic scales (Fig. 1 1a). However, in a finite region around half-filling, disorder induces phase separation into macroscopic regions of high and low density (Fig. If b). We give approximate arguments to understand the origin and nature of this phase separation, and to obtain the form of the phase diagram in the current-density plane. This sort of behaviour has also been seen earlier in a model with a single weak bond [11]. We argue that disorder-induced phase separation is a generic feature of systems in which the current $J$ versus density $\rho$ shows a maximum at some intermediate density, in the absence of disorder.

In the version of the DASEP in which the easy direction of hopping is itself a quenched random variable, the model represents a system of hard-core particles in a random potential with an overall downward tilt, but with backbends of arbitrary length. Long backbends severely limit the maximum current that can flow through the system, and in fact the current decreases to zero as the system size increases (Fig. 2); the system is in the vanishingcurrent regime.

Although our emphasis in this paper is on the analysis of lattice models, we comment briefly on certain constraints that are important in a continuum description. Such a description is expected to be valid for the largescale, long-time behaviour, and is based on stochastic differential equations involving appropriate coarse-grained variables. It is argued that quenched randomness is manifest in random multiplicative coefficients in a gradient expansion. Conservation of particle number - which implies spatial constancy of the current in the steady state - imposes strong constraints on these terms.

In one dimension, using a well known mapping [12], the particle models are equivalent to stochastic growth models of a $1-d$ interface moving in a $2-d$ medium. The interface moves with a speed proportional to the cur- 
rent in the particle model. The disordered jump rates now become local growth rates which are disordered in a columnar fashion for the moving interface [13]. The three principal regimes of behaviour discussed above for the particle models translate into distinct regimes for interface motion, namely (i) a moving interface with normal roughness, (ii) a moving interface with large segments with different mean slopes, and (iii) an interface with different-slope segments, which is stationary in the thermodynamic limit.

The paper is organized as follows. In Section [1] we define and discuss the steady state properties of the disordered drop-push process in arbitrary dimensionality. The disordered asymmetric exclusion process with only forward-easy-direction of hopping, but quenched random rates, is discussed in Section III; the case in which there are some backward-easy-direction bonds is discussed in Section IV. In Section V we discuss the constraints on a continuum description, while Section V1 discusses the implications of our results for models interface growth in the presence of columnar disorder. Section VII is the conclusion.

\section{DISORDERED DROP-PUSH PROCESS : DDPP}

The drop-push process was initially introduced in [6,10] as a model of activated flow involving transport through a series of traps of equal depths. The dynamics consists of activated hops together with a cascade of overflows following each move. The disordered version of the model may be considered as a discrete model of activated fluid flow down an inclined rugged slope with lakes of varying depths; see Figs. 3, 1. This is similar to above-threshold behaviour of the model considered in [14. In this section we show that the steady state and current can be found exactly in all dimensions for the DDPP and its generalizations.

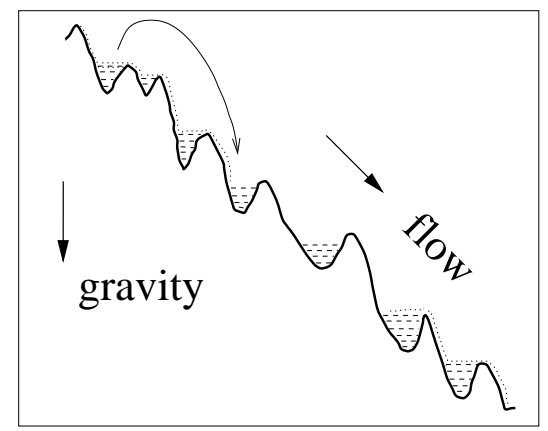

FIG. 3. Schematic diagram of water flowing down a rugged hill-side. Water from a lake higher up cascades downhill, under the action of gravity, until it finds a partially filled lake. The unequal capacities of the lakes are the quenched variables in the system.

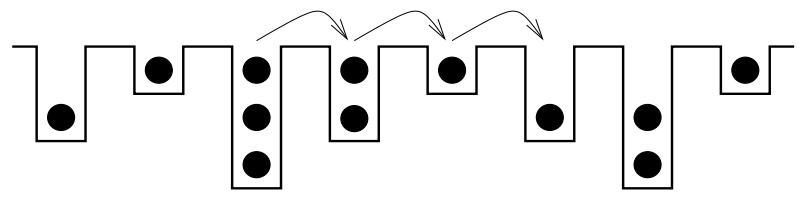

FIG. 4. A Disordered Drop-Push Process (DDPP) configuration and move in $d=1$.

\section{A. The model}

The model in $d$-dimensions is defined on a hypercubic lattice with periodic boundary conditions along all the $d$ axes (with unit vectors $\left\{\hat{e}_{\nu} \mid \nu=1 \cdots d\right\}$ ). At each site $r$ is a well which can hold at most $l_{r}$ particles (Figs. (4.5) with $l_{r}$ 's chosen independently from some probability distribution $P(l)$. The configuration $\mathcal{C}$ of the system is specified by specifying the set of occupation numbers $\left\{n_{r}\right\}$ with $\left(0 \leq n_{r} \leq l_{r}, \forall r\right)$. Further, with each site $r$ is assigned a set $\left\{\epsilon\left(n_{r} \mid l_{r}\right) ; n_{r}=1, \cdots, l_{r}\right\}$ of positive random numbers chosen from some given distribution [15]. The dynamics is stochastic. In a time interval $\overrightarrow{d t}$, with a probability $p_{ \pm \nu} \epsilon\left(n_{r} \mid l_{r}\right) d t$, the topmost particle in the well $r$ hops out, and drops into well $r \pm \hat{e}_{\nu}$, i.e. into the adjacent well in the $\pm \nu$ th direction. Here $\left\{p_{ \pm \nu} ; \nu=1, . ., d\right\}$ are a set of site-independent positive numbers satisfying $\sum_{\nu=1}^{d}\left(p_{\nu}+p_{-\nu}\right)=1$. Now, if well $r \pm \hat{e}_{\nu}$ is already full, then the particle gets pushed further preserving the direction of the initial jump to the next site and so on. The cascade of transfers terminates once a partially full well is encountered. Note that here the set of jump-rates $\left\{\epsilon\left(n_{r} \mid l_{r}\right)\right\}$ are site-dependent as well as functions of the occupation numbers. These rates, together with the welldepths $\left\{l_{r}\right\}$, constitute the quenched random variables in the model. The set of probabilities $\left\{p_{ \pm \nu}\right\}$ determines the direction of the global bias $\vec{E}=\sum_{\nu=1}^{d}\left(p_{\nu}-p_{-\nu}\right) \hat{e}_{\nu}$ and, as will be shown in Section IIB, also the direction and magnitude of the steady state current in the model. However, they do not enter the expression for the normalized invariant measure.

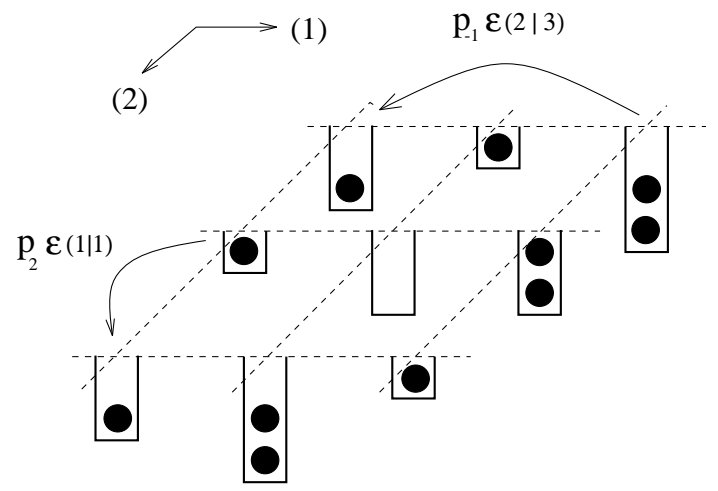

FIG. 5. The DDPP model in $d=2$. The model can be generalized to $d>2$ (see text). The rates $\epsilon\left(n_{r} \mid l_{r}\right)$ depend on the well depth $l_{r}$ as well as the occupation number $n_{r}$. 
Though all the results we will discuss holds for any arbitrary choice of the $\epsilon$ 's, in a physical system they should be determined from the details of the trapping mechanisms etc., e.g. they may be taken to be of the Kramers form $\epsilon\left(n_{r} \mid l_{r}\right) \propto \exp \left[-g\left(l_{r}-n_{r}\right)\right]$ for situations where the jumps are activated [16].

\section{B. Invariant measure}

The time evolution of the probability $\mathcal{P}(\mathcal{C})$ for the system to be in configuration $\mathcal{C}$ is given by the master equation 17]

$$
\frac{d}{d t} \mathcal{P}(\mathcal{C})=\sum_{\mathcal{C}^{\prime \prime}} W\left(\mathcal{C}^{\prime \prime} \rightarrow \mathcal{C}\right) \mathcal{P}\left(\mathcal{C}^{\prime \prime}\right)-\sum_{\mathcal{C}^{\prime}} W\left(\mathcal{C} \rightarrow \mathcal{C}^{\prime}\right) \mathcal{P}(\mathcal{C})
$$

Here the $W$ 's are the transition matrix elements identified with the rates $\epsilon^{\prime}$ s defined in the model. e.g. if the transition $\mathcal{C} \rightarrow \mathcal{C}^{\prime}$ involves moving the topmost of the $n_{r}$ particles at $r$ to $r^{\prime}$ along the $\nu$ th direction, then $W\left(\mathcal{C} \rightarrow \mathcal{C}^{\prime}\right)=p_{\nu} \epsilon_{r}\left(n_{r} \mid l_{r}\right)$. The steady-state or the invariant measure of the dynamics is the set of time independent weights $\{\mu(\mathcal{C})\}$ satisfying (11) above. Hence the problem of finding the invariant measure reduces to that of finding a set of positive weights $\{\mu(\mathcal{C})\}$ such that the total incoming flux into any configuration $\mathcal{C}$ (the first sum in (11)) equals the total flux out of $\mathcal{C}$ (the second sum in (11)). The uniqueness of the invariant measure is ensured by the connectedness property of the $W$-matrix, i.e. every configuration can be reached from any other by a sequence of transitions [17].

We claim that the (unnormalized) measure of configuration $\mathcal{C}\left(\left\{n_{r}\right\}\right)$ in the steady state has the product form

$$
\mu(\mathcal{C})=\prod_{r} u_{r}\left(n_{r}\right)
$$

Here $u_{r}$ are the single-site weights defined as

$$
u_{r}\left(n_{r}\right)= \begin{cases}1 & \text { if } n_{r}=0 \\ \tau_{r}(1) \cdots \tau_{r}\left(n_{r}\right) & \text { if } 0<n_{r} \leq l_{r}\end{cases}
$$

where $\tau_{r}\left(n_{r}\right)=\epsilon_{0} / \epsilon\left(n_{r} \mid l_{r}\right)$ with $\epsilon_{0}$ being a microscopic rate.

To show that (2) is indeed the invariant measure for the DDPP we show that it is possible to associate configuration $\mathcal{C}^{\prime \prime}$ in one-to-one correspondence with every $\mathcal{C}^{\prime}$ obtained from $\mathcal{C}$ by an elementary transition such that

$$
W\left(\mathcal{C} \rightarrow \mathcal{C}^{\prime}\right) \mu(\mathcal{C})=W\left(\mathcal{C}^{\prime \prime} \rightarrow \mathcal{C}\right) \mu\left(\mathcal{C}^{\prime \prime}\right)
$$

The above is the condition of pairwise balance [10] which ensures that the terms in the two sums on the right hand side of (11) cancel in pairs. Pairwise balance has been used earlier to find steady states of translationally invariant systems [10,18. We now see that it can be used effectively to deduce the steady state of a disordered system as well.

Suppose the transition $\mathcal{C} \rightarrow \mathcal{C}^{\prime}$ involves hopping a particle at site $r$ to a site $r^{\prime}=r+\Delta r^{\prime} \hat{e}_{\nu}$ with all wells in between along the $\nu$ th axis full (Fig. 6). Also suppose the well $r^{\prime \prime}=r-\Delta r^{\prime \prime} \hat{e}_{\nu}$ is not full but all wells between $r^{\prime \prime}$ and $r$ are full. The configuration $\mathcal{C}^{\prime \prime}$ is constructed such that it is identical to $\mathcal{C}$ at all sites except at the sites $r^{\prime \prime}$ and $r$, at which $n_{r^{\prime \prime}}^{\prime \prime}=n_{r^{\prime \prime}}+1, n_{r}^{\prime \prime}=n_{r}-1$. With $\mathcal{C}^{\prime \prime}$ thus defined, the pairwise balance condition (4)

$$
\epsilon\left(n_{r} \mid l_{r}\right) \prod_{r} u_{r}\left(n_{r}\right)=\epsilon\left(n_{r^{\prime \prime}}^{\prime \prime} \mid l_{r^{\prime \prime}}\right) \prod_{r} u_{r}\left(n_{r}^{\prime \prime}\right)
$$

reduces to

$$
\epsilon\left(n_{r} \mid l_{r}\right) u_{r^{\prime \prime}}\left(n_{r^{\prime \prime}}\right) u_{r}\left(n_{r}\right)=\epsilon\left(n_{r^{\prime \prime}}^{\prime \prime} \mid l_{r^{\prime \prime}}\right) u_{r^{\prime \prime}}\left(n_{r^{\prime \prime}}^{\prime \prime}\right) u_{r}\left(n_{r}^{\prime \prime}\right),
$$

since $\mathcal{C}$ and $\mathcal{C}^{\prime \prime}$ differ only at the sites $r^{\prime \prime}$ and $r$. As can be explicitly checked, condition (6) is satisfied in view of the form of the weights $u_{r}\left(n_{r}\right)$. The prescription above ensures one-to-one correspondence between configurations $\mathcal{C}^{\prime \prime}$ and $\mathcal{C} \rightarrow \mathcal{C}^{\prime}$ transitions.

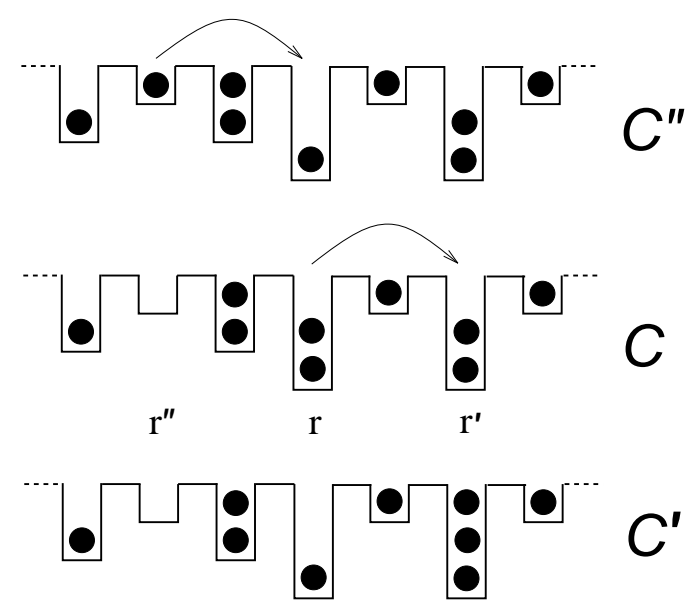

FIG. 6. Construction of configurations satisfying the pairwise balance condition in $d=1$.

In the limit of large $L$ we use the grand canonical description and write the normalized probability of configuration $\mathcal{C}$ in the steady state as

$$
\mathcal{P}(\mathcal{C})=\frac{z^{N_{P}} \mu(\mathcal{C})}{\prod_{r} Z_{r}}
$$

where the site generating functions are given by

$$
Z_{r}=\sum_{n_{r}=0}^{l_{r}} u_{r}\left(n_{r}\right) z^{n_{r}}
$$

and $N_{p}$ is the number of particles in the configuration. Here $z$ is the fugacity and will be shown to be directly related to the steady-state current. The mean particle density at site $r$ is given as $\left\langle n_{r}\right\rangle=z \partial \ln Z_{r} / \partial z$ [19]. Thus 
the fugacity $z$ is related to the mean particle density $\rho$ of the system through

$$
\rho=\frac{1}{L^{d}} \sum_{r}\left\langle n_{r}\right\rangle=\frac{z}{V} \sum_{r} \frac{\partial \ln Z_{r}}{\partial z}
$$

The steady state is characterized by a spatially uniform $z$ but inhomogeneous site densities.

\section{Steady-state current}

For simplicity of presentation, we first derive a closed form expression for the steady-state current $J_{0}$ for the 1- $d$ fully asymmetric model. Then we use these results to write down the current for the general $d$-dimensional case.

(i) $d=1$, with forward jumps: In this case, for notational convenience, we replace the lattice site index $r$ by a single integer index $i$. Further, we allow jumps only in one direction $\left(\nu=1: p_{1}=p, p_{-1}=0\right)$. For an infinite system we write the current $J_{i, i+1}$ in the bond $(i, i+1)$ as

$$
\begin{aligned}
J_{i, i+1}= & \sum_{j<i} \sum_{n_{j}=1}^{l_{j}} p \epsilon\left(n_{j} \mid l_{j}\right) P_{j}\left(n_{j}\right) \prod_{j<k \leq i} P_{k}\left(l_{k}\right) \\
& +\sum_{n_{i}=1}^{l_{i}} p \epsilon\left(n_{i} \mid l_{i}\right) P_{i}\left(n_{i}\right)
\end{aligned}
$$

where

$$
P_{i}\left(n_{i}\right)=u_{i}\left(n_{i}\right) z^{n_{i}} / Z_{i}
$$

is the probability that site $i$ has occupancy $n_{i}$. Note that the product form of the measure (2) has been exploited to write the above expression with decoupled joint probabilities.

It is cumbersome, and not very instructive, to perform the sum in (10) to obtain the current in a closed form. Instead we use the spatial constancy of the current in the steady state to arrive at the result. We note that in the above expression the first term represents contribution of jumps originating from site $j$ to the left of site $i$ with all sites in between full. The second sum represents jumps from the site $i$ itself contributing to $J_{i, i+1}$. The first sum may be rewritten as

$$
\begin{aligned}
P_{i}\left(n_{i}\right)[ & \sum_{j<i-1} \sum_{n_{j}=1}^{l_{j}} \epsilon\left(n_{j} \mid l_{j}\right) P_{j}\left(n_{j}\right) \prod_{j<k \leq i-1} P_{k}\left(l_{k}\right) \\
& \left.+\sum_{n_{i-1}=1}^{l_{i-1}} \epsilon\left(n_{i-1} \mid l_{i-1}\right) P_{i-1}\left(n_{i-1}\right)\right] .
\end{aligned}
$$

The quantity within square brackets [.] is immediately recognised to be $J_{i-1, i}$ by comparing it with (10). Physically, this means that all jumps contributing to $J_{i-1, i}$, for which site $i$ is completely full, also contribute to $J_{i, i+1}$. Hence we have the recursion

$$
J_{i, i+1}=P_{i}\left(l_{i}\right) J_{i-1, i}+\sum_{n_{i}=1}^{l_{i}} p \epsilon\left(n_{i} \mid l_{i}\right) P_{i}\left(n_{i}\right)
$$

relating $J_{i, i+1}$ to $J_{i-1, i}$. Now, since $\left\{P_{i}\left(n_{i}\right)\right\}$ are the steady-state site probabilities, and since in the steady state all the bond currents must be equal (i.e. $J_{i, i+1}=$ $\left.J_{i-1, i}=\ldots . .=J_{0}\right)$, from (12) we obtain

$$
J_{0}=\frac{\sum_{n_{i}=1}^{l_{i}} \epsilon\left(n_{i} \mid l_{i}\right) P_{i}\left(n_{i}\right)}{1-P_{i}\left(l_{i}\right)}=p \epsilon_{0} z
$$

where (22) and (11) have been used in the second step above.

Note that the steady-state current does not depend upon the detailed spatial arrangement of wells. It is only a function, through the fugacity $z$, of the density and the total number of wells of different types in a particular realization of disorder.

(ii) $d=1$, with jumps in both directions: We can write the current $J_{i, i+1}$ in bond $(i, i+1)$ as the difference between the current $J_{i, i+1}^{r}$ due to the rightward jumps and the current $J_{i, i+1}^{l}$ due to the leftward jumps. This can be done since in each cascade the direction of the initial jump is preserved. Now, we use the result for the fully asymmetric case above to each of these currents separately to obtain $J_{i, i+1}=J_{0}=\left(p_{1}-p_{-1}\right) \epsilon_{0} z$

(iii) $d>1$ : To generalize the above results to $d>1$ we note that for DDPP in $d>1$, the invariant measure (2) is the same if we single out a particular direction, say $\nu$, and allow jumps only along that direction. Together with the direction preservation of individual jumps, this allows us to write the expression for the current in any dimension:

$$
\overrightarrow{J_{0}}=\left[\sum_{\nu=1}^{d}\left(p_{\nu}-p_{-\nu}\right) \hat{e}_{\nu}\right] \epsilon_{0} z=\epsilon_{0} z \vec{E}
$$

where $\vec{E} \equiv \sum_{\nu=1}^{d}\left(p_{\nu}-p_{-\nu}\right) \hat{e}_{\nu}$ is the external drive. As in the $d=1$ case, the magnitude and the direction of the steady-state current does not depend upon the detailed arrangements of the wells.

\section{Static two-point correlation functions}

Because of the product form of the measure, the connected part of the equal-time density-density correlation function

$$
G_{r}(\Delta r)=\left\langle n_{r} n_{r+\Delta r}\right\rangle-\left\langle n_{r}\right\rangle\left\langle n_{r+\Delta r}\right\rangle
$$

vanishes identically for $\Delta r \neq 0$. Consequently, the fluctuation of the number of particles in $r$ consecutive sites along a straight line can be computed exactly:

$$
\begin{aligned}
\Gamma_{i}^{2}(r) & =\left\langle\left[\sum_{j=i+1}^{i+r}\left(n_{j}-\left\langle n_{j}\right\rangle\right)\right]^{2}\right\rangle \\
& =\sum_{j=i+1}^{i+r}\left(z \frac{\partial}{\partial z}\right)^{2} \ln Z_{j} .
\end{aligned}
$$


The second step follows from the product form of the measure.

For $d=1$, a standard mapping discussed in Section VI introduces height variables defined by

$$
h_{i}=\sum_{j \leq i} 2\left(\left\langle n_{j}\right\rangle-n_{j}\right)
$$

Evidently, $\Gamma_{i}^{2}(r)$ is the equal-time height-height correlation $\left\langle\left(h_{i+r}-h_{i}\right)^{2}\right\rangle$. Averaging over the disorder distribution gives $\overline{\Gamma^{2}}(r) \sim r$ implying that the 'roughness' exponent $\alpha$ (defined by $\overline{\Gamma^{2}}(r) \sim r^{2 \alpha}$ ) is $1 / 2$.

\section{E. Two-rate DDPP model: Explicit results}

Let us consider a drop-push model where the maximum occupancy of each site is restricted to one, i.e. $l_{r}=1, \forall r$, but the hopping rates $\epsilon_{r}(1)$ are disordered, and chosen independently from the binary distribution

$$
\operatorname{Prob}\left(x=\epsilon_{a}\right)=1-f, \quad \operatorname{Prob}\left(x=\epsilon_{b}\right)=f .
$$

This model has the essential ingredients of disorder present in the original DDPP, yet it is simple enough that explicit, closed form relations between the mean density $\rho$ and fugacity $z$, and hence the steady-state current $\overrightarrow{J_{0}}$, can be written down.

Let us denote by $Z_{a}$ and $Z_{b}$ the site generating functions for the $a$ and the $b$ sites respectively. Using (8) and (2), these are given by $Z_{a}=1+\epsilon_{0} z / \epsilon_{a}$ and $Z_{b}=$ $1+\epsilon_{0} z / \epsilon_{b}$. Now, since the fractions of $a$ and $b$ sites are $1-f$ and $f$ respectively, (9) reduces to

$$
\rho=(1-f) \frac{\epsilon_{0} z}{\epsilon_{a}+\epsilon_{0} z}+f \frac{\epsilon_{0} z}{\epsilon_{b}+\epsilon_{0} z}
$$

This can be easily inverted to obtain $z$ as a function of $\rho$, e.g. for $f=1 / 2$ and $\epsilon_{a}=\epsilon_{0}=\epsilon_{b} / q$ we obtain

$z(\rho)=\frac{\sqrt{(1-q)^{2}(1 / 2-\rho)^{2}+q}-(1+q)(1 / 2-\rho)}{2(1-\rho)}$

Since $z(\rho)$ is known, the steady-state current is trivially obtained from (14).

Finally, the correlation function $\Gamma_{i}^{2}(r)$ of (16), upon disorder averaging, may be written as

$$
\begin{aligned}
\overline{\Gamma^{2}}(r) & =r\left(z \frac{\partial}{\partial z}\right)^{2}\left[(1-f) \ln Z_{a}+f \ln Z_{b}\right] \\
& =\frac{1}{2}\left[\frac{z}{(1+z)^{2}}+\frac{q z}{(q+z)^{2}}\right] r
\end{aligned}
$$

where $z$ is given by $(20)$ above.

\section{F. Generalized Disordered Drop-push Process: GDDP}

We may consider a generalized version of the droppush process in which, in addition to the particle moves, independent hole moves are also allowed. For simplicity we restrict ourselves to the generalized version of the single occupancy DDPP introduced above. This generalized model may be regarded as the disordered lattice gas analogue of the Toom interface dynamics in the low-noise limit [20]; see Section VI. The techniques developed for the DDPP may be used to obtain the exact steady-state measure and other quantities such as current and static correlations provided a certain condition [(22) below] is met.

The model in $d$ dimensions is defined on a hypercubic lattice with periodic boundary conditions along all the $d$ axes (with unit vectors $\left\{\hat{e}_{\nu} \mid \nu=1 \cdots d\right\}$ ), similar to the DDPP. Each site $r$ of the lattice can hold either a particle $\left(n_{r}=1\right)$ or a hole $\left(n_{r}=0\right)$. The configuration $\mathcal{C}$ of the system is specified by specifying the occupation number of each well $\left\{n_{r}\right\}$ with $\left(n_{r} \in\{0,1\}, \forall r\right)$. Further, to each site $r$ is assigned a pair of positive random numbers $\left(\alpha_{r}, \beta_{r}\right)$ chosen from some distribution. The dynamics is stochastic and is very similar to that for the DDPP dynamics: in a time interval $d t$, a particle at site $r$ is exchanged with the closest hole in the $\pm \nu$ th direction with a probability $p_{ \pm \nu} \alpha_{r} d t$ (Fig. 7). For identical particles this move is equivalent to a cascade of particle moves terminating at the first vacant site as in the drop-push dynamics. Likewise, in interval $d t$, a hole at site $r$ is exchanged with the closest particle along the $\nu$ th direction with probability $q_{\nu} \beta_{r} d t$. This can be looked upon of as a cascade of hole-moves analogous to the cascade of particle moves. Here, as in the DDPP, the $p_{\nu}$ 's and $q_{\nu}$ 's are all non-negative and satisfy $\sum_{\nu=1}^{d}\left(a_{\nu}+a_{-\nu}\right)=1 ; a=p, q$. Further we chose $\alpha$ 's and $\beta$ 's such that

$$
\alpha_{r} \beta_{r}=K
$$

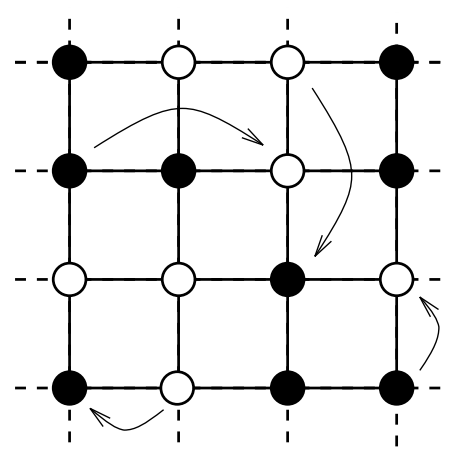

FIG. 7. Generalized Disordered Drop-push Process (GDDP) configuration and moves in $d=2$. 
where $K$ is a constant independent of $r$. As we will see below, this particular choice of the jump-rates allows the exact determination of the invariant measure of the model. Physically this choice is quite reasonable since it implies that the sites which act as traps for particles (low $\alpha_{r}$ ) are more transparent to holes (high $\beta_{r}$ ) and vice versa. As pointed out earlier the non-disordered version of this model, i.e. $\left\{\alpha_{r}=\alpha, \beta_{r}=\beta, \forall r\right\}$ in $1-d$, is the lattice gas equivalent of the low-noise driven Toom interface dynamics [20].

The master equation (11) governing the time evolution of the system now includes terms corresponding to hole moves as well. Since each micro-step involves either only particle moves or hole moves, we use the principle of pairwise balance for the particle moves and hole moves separately. We work in the grand canonical picture in the thermodynamic limit.

If only particle moves were allowed the invariant measure would be given by

$$
\mu_{\text {pcle }}(\mathcal{C})=\prod_{r} u_{r}\left(n_{r}\right)
$$

The single site weights $u_{r}$ are given by

$$
u_{r}\left(n_{r}\right)= \begin{cases}1 & \text { if } n_{r}=0 \\ \epsilon_{0} / \alpha_{r} & \text { if } n_{r}=1\end{cases}
$$

Introducing the fugacity $z$ and site generating functions $Z_{r}=1+z u_{r}(1)$ we can write the normalized single site probabilities as $P_{r}^{p c l e}(0)=1 / Z_{r}$ and $P_{r}^{p c l e}(1)=$ $z u_{r}(1) / Z_{r}$.

Similarly, with only the hole moves, the invariant measure has the product form

$$
\mu_{\text {hole }}(\mathcal{C})=\prod_{r} v_{r}\left(n_{r}\right)
$$

where $n_{r}=1(0)$ refers to the presence (absence) of a hole. The single site weights $v_{r}$ are given by

$$
v_{r}\left(n_{r}\right)=\left\{\begin{array}{ll}
1 & \text { if } n_{r}=0 \\
\epsilon_{0} / \beta_{r} & \text { if } n_{r}=1
\end{array} .\right.
$$

Introducing the fugacity $y$ for holes and site generating functions $Y_{r}=1+y v_{r}(1)$ we can write the normalized single-site probabilities as: $P_{r}^{\text {hole }}(0)=1 / Y_{r}$ and $P_{r}^{\text {hole }}(1)=y v_{r}(1) / Y_{r}$.

Now, since each site is occupied either by a particle or a hole, we must have $P_{r}^{\text {pcle }}(0)=P_{r}^{\text {hole }}(1)$ and $P_{r}^{\text {pcle }}(1)=P_{r}^{\text {hole }}(0)$. Using the detailed forms of $P_{r}^{\text {pcle }}$ ' $\mathrm{s}$ and $P_{r}^{\text {hole }}$ 's, we arrive at the condition (22) with $K \equiv$ $\epsilon_{0}^{2} y z$. If this condition is satisfied then the invariant measure for GDDP is given by either (23) or (25), since both are equivalent.

In a similar manner as for the DDPP the current due to the particle moves and hole moves may be computed. The total particle current due to both types of moves, in $d$ dimensions, is given by

$$
\overrightarrow{J_{0}}=\epsilon_{0} z \sum_{\nu=1}^{d}\left(p_{\nu}-p_{-\nu}\right) \hat{e}_{\nu}-\epsilon_{0} y \sum_{\nu=1}^{d}\left(q_{\nu}-q_{-\nu}\right) \hat{e}_{\nu}
$$

As for the DDPP, static density-density correlations in the steady state vanish identically on account of the product form of the steady-state measure. In $d=1$ the height-height correlation is given by (16).

\section{DISORDERED ASYMMETRIC SIMPLE EXCLUSION PROCESS: DASEP}

The asymmetric simple exclusion process (ASEP) is a prototype model for studying nonequilibrium phenomena in the context of lattice gases [21,22]. When discussing the effect of quenched disorder, it is important to distinguish between cases in which (a) the easy direction of hopping in each bond is the same but hopping rates are random, and (b) the easy direction is itself a random variable. The latter case is studied in Section IV. In this section, we consider a 1- $d$ system with disorder of type (a) and show that quenched disorder can induce macroscopic phase separation. Using a variety of arguments we sketch the phase coexistence curve in the current $\left(J_{0}\right)$ mean density $(\rho)$ plane. This agrees qualitatively with the results obtained from the Monte Carlo (MC) simulations.

\section{A. The model}

In one dimension, we define the disordered asymmetric simple exclusion process on a ring of $L$ sites. Each site can hold either 1 or 0 particle. To each bond $(i, i+1)$ of the lattice is assigned a quenched random rate $\alpha_{i, i+1}$ chosen independently from some probability distribution $\operatorname{Prob}(\alpha)$. The dynamics is stochastic: in a time interval $d t$ a particle at site $i$ attempts to hop, with probability $p \alpha_{i, i+1} d t$, to site $i+1$. The move is completed if and only if site $i+1$ is unoccupied (see Fig. 8).

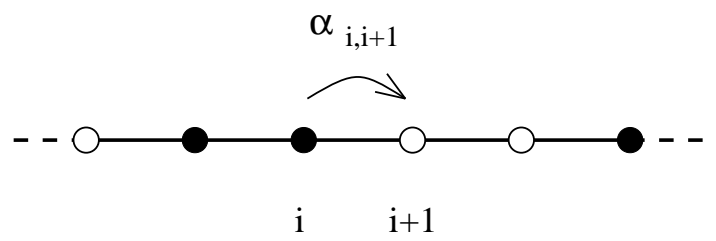

FIG. 8. The disordered fully asymmetric simple exclusion process (DASEP) and moves in $d=1$.

For the model defined above no analytically exact characterization of the steady-state measure could be obtained. A simpler model in which there is only one defect bond has been studied in detail by Janowsky and Lebowitz [11, but in this case too the exact steady-state measure is not known. We use Monte Carlo simulations and a mean-field approximation to demonstrate some striking effects of quenched disorder. 


\section{B. Current-density plot and density profile in steady state}

Figure 9 shows the steady-state current $J_{0}$ vs mean density $\rho$ plot, obtained from MC simulations, for a system of size $L=8000$ and the rates $\alpha$ chosen from the binary distribution

$$
\operatorname{Prob}(\alpha=r)=f, \quad \operatorname{Prob}(\alpha=1)=1-f .
$$

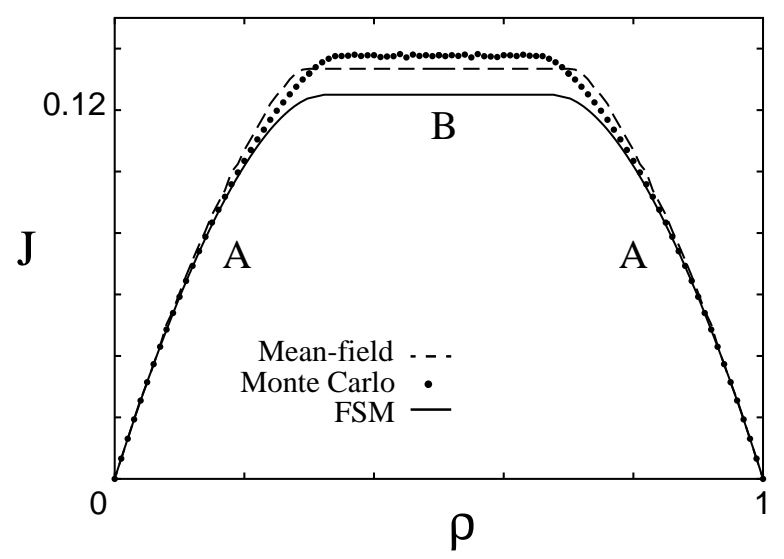

FIG. 9. The current-density plot for the DASEP for a given realization of disorder for a system of size $L=8000$. The hopping rates are chosen from the distribution (28) with $r=f=1 / 2$. The filled circles are the MC results and the dashed line is the mean field curve. The solid line represents the $J-\rho$ curve for the Fully Segregated Model (FSM) for the same values of the parameters.

Here $f$ is the fraction of weak bonds, and $r$ is a measure of the strength of the weak bonds. We used the values $r=1 / 2, f=1 / 2$ in our numerical work. For a specified mean density $\rho$, a random initial distribution of $N_{p}=\rho L$ particles on $L$ sites is chosen and the system is allowed to settle into a steady state by evolving it for a sufficiently large number of MC steps. Then the current across each bond is obtained by counting the total number of jumps across that bond, over a large number of MC steps. An average of all the bond currents thus computed is taken as $J_{0}$, as currents across all bonds are equal in the steady state. $J_{0}$ is a symmetric function of density around $\rho=1 / 2$ as a result of a certain symmetry with respect to particle-hole interchange (see Appendix A). As may be expected, the current for the disordered system lies between the corresponding values of the two pure reference systems with $r=1$ and $r=1 / 2$ on all the bonds. The more striking qualitative difference between the disordered and pure systems is the appearance of a plateau (Regime B in Fig. 9) for a range of densities $|\rho-1 / 2| \leq \Delta$. In this regime, the current is independent of the mean density and equals the maximum allowed current in the system. The approximate size $\Delta$ of regime
$\mathrm{B}$, which is a function of $r$ and $f$, is obtained in subsection IIIE below.

We studied the steady state density profiles characterized by the set of site densities $\left\{\rho_{i} \equiv\left\langle n_{i}\right\rangle\right\}$ in both regimes $\mathrm{A}$ and $\mathrm{B}$, using $\mathrm{MC}$ simulations. We found that in regime $\mathrm{A}$ the system is homogeneous on a macroscopic scale, while in regime B it shows macroscopic density segregation. Figure 10 shows the steady state density profiles for three representative mean densities - one from regime $\mathrm{A}$ and two from regime $\mathrm{B}$. Evidently there is a large qualitative difference between the profiles in the two regimes. In regime A (Fig. 10a), there are density variations (shocks) only over microscopic scales; coarsegraining over a few lattice spacings leads to a spatially uniform density. In contrast to this, in the profiles corresponding to regime B (Fig. 10b), there are density inhomogeneities over length scales comparable to the system size $L$, in addition to the shocks on a microscopic scale. This segregation into high and low density phases, with large shocks separating them, is reminiscent of phase separation, and occurs over the full range of mean particle densities corresponding to regime B. A qualitatively similar phenomenon has been found in a system with one defect bond, studied in 11].

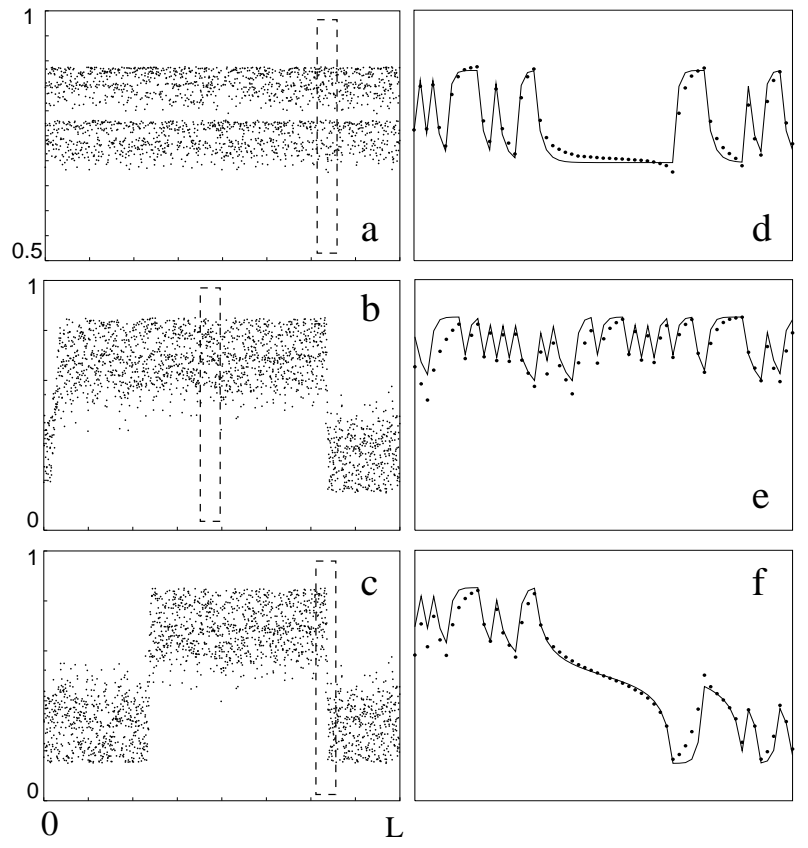

FIG. 10. Density profiles for the DASEP for a system of size $L=8000$ for a given realization of disorder at three fillings (a) $\rho=0.8$, (b) $\rho=0.6$, and (c) $\rho=0.5$. In $\mathrm{d}$,e and $\mathrm{f}$ are shown the blow-ups of the regions enclosed in the dashed boxes in a,b and c respectively. Circles are MC profiles and the continuous lines are mean-field results.

The number of different large-scale regions of high and low density shows fluctuations from one realization of disorder to another. As the size of the system is increased, we monitored the mean number of these regions, and 
found that it is nearly constant, or perhaps increases very mildly - certainly, much less fast than linearly in the size of the system. This implies that the characteristic length scale of density segregation increases indefinitely in the thermodynamic limit.

\section{Mean-field approximation}

We now turn to a mean-field approximation, which assumes no correlations between site densities in the steady state. We will see that it captures most of the steadystate features found in the MC simulations above, including the steady-state density profile.

The time-averaged steady-state current $J_{i, i+1}$ in the bond $(i, i+1)$ is given by $J_{i, i+1}=\alpha_{i, i+1}\left\langle n_{i}\left(1-n_{i+1}\right)\right\rangle$. In view of the mean-field approximation $\left\langle n_{i} n_{j}\right\rangle=\left\langle n_{i}\right\rangle\left\langle n_{j}\right\rangle$ this reduces to

$$
J_{i, i+1}=\alpha_{i, i+1}\left\langle n_{i}\right\rangle\left\langle 1-n_{i+1}\right\rangle
$$

To compute the steady-state current $J_{0}$ as a function of the mean particle density $\rho$ for a given realization of disorder, we use the two iteration schemes described below, which yield equivalent results.

(i) Constant current iteration scheme. For a given system of size $L$ and for a fixed value $J_{0}$ for the current, we iterate the following set of equations

$$
\rho_{i+1}=1-J_{0} /\left(\alpha_{i, i+1} \rho_{i}\right) \quad i=1, . ., L
$$

around the chain starting with, say, some value $\rho_{1}$ (periodic boundary conditions imply $\rho_{i+L}=\rho_{i}$ ). If $J_{0}$ is less than a certain value $J_{\max }^{M F}$, which is the maximum current supported by the system within the mean-field approximation, then the iteration scheme converges, i.e. we get all the site densities in the physically acceptable range $[0,1]$. The average of these site densities gives the mean density of the system corresponding to the stationary current $J_{0}$. There are in general two values of the mean particle density corresponding to an allowed value of $J_{0}$ and the iteration scheme converges to one or the other depending on the initial value of the density $\rho_{1}$.

However, in this scheme we find that the number of iterations required for convergence increases without bound, as the trial value $J_{0}$ gets closer to $J_{\max }^{M F}$ from below. This divergence of the iteration scheme is presumably due to the existence of the plateau in the $J v s \rho$ curve, i.e. there exist many values of $\rho$ for $J_{0}$ very close to $J_{\max }^{M F}$. Hence to obtain the density profile for $\rho$ in the density segregated regime we resort to the constant density iteration scheme described below.

(ii) Constant density iteration scheme. In this scheme we begin by assigning site densities $\left\{0 \leq \rho_{i}(0) \leq 1\right\}$ to the lattice sites subjected to the constraint $\frac{1}{L} \sum_{i} \rho_{i}(0)=\rho$. The site densities are updated in parallel according to:

$$
\rho_{i}(t+1)=\rho_{i}(t)+J_{i-1, i}(t)-J_{i, i+1}(t) ; \quad i=1, . ., L
$$

where $J_{i, i+1}(t)=\alpha_{i, i+1} \rho_{i}(t)\left[1-\rho_{i+1}(t)\right]$ in view of (29).

We refer to this as the constant density scheme, since in each iteration the total density remains unchanged, i.e $\sum_{i} \rho_{i}(t+1)=\sum_{i} \rho_{i}(t)$. After a sufficient number of iterations, which depends upon the starting mean density $\rho$, the set of site densities converge to a set of numbers $\left\{\rho_{i}\right\}$ and the current on each bond converges to the steady state current $J_{0}$.

The steady-state density profiles and the $J_{0}$ vs $\rho$ plot $(0 \leq \rho \leq 1)$ for a given configuration of disorder obtained using these schemes is shown in Figs. 10 and 9 respectively. It is evident that the mean-field approximation (29) reproduces quite well not only the $J-\rho$ relationship, but also the density profile, including the locations of shocks, though not the shapes of individual shocks.

\section{Qualitative explanation of phase separation}

Although the mean-field approximation of the previous subsection successfully reproduces many features in the steady state, it does not yield a simple understanding of the phase separation (Fig. 10) or the plateau in the $J-\rho$ curve (Fig. 9) in terms of the macroscopic parameters of the model. We conjecture that underlying the behaviour of the DASEP in different regimes is a Maximum Current principle: For a given mean density, the system settles into a steady-state which maximizes the stationary current. Such a maximum current principle has been used to describe phase separation in the asymmetric simple exclusion process with open boundary conditions in 23 .

To use the maximum-current principle to have a qualitative understanding of the phase separation in DASEP, let us assume that the density in each stretch of like bonds is uniform. This approximation is in fact exact in the Fully Segregated Model (FSM) discussed in the following subsection. Let us denote stretches of $\alpha=1$ bonds by $\mathrm{X}$ and stretches of $\alpha=r<1$ bonds by $\mathrm{Y}$. The two parabolas in Fig. 11 are the steady-state $J$ vs $\rho$ curves for the two pure reference systems all $\mathrm{X}$ and all $\mathrm{Y}$. In the disordered system, since the steady-state current has to be spatially constant, the possible densities are given by the four intersections of the line $J=J_{0}$ with the two parabolas. If the mean density is in the range $\rho \leq 1 / 2-\Delta($ or $\rho \geq 1 / 2+\Delta)$, then the allowed densities for the $\mathrm{X}$ and $\mathrm{Y}$ stretches are $\rho_{1}, \rho_{2}$ (or $\left.\rho_{4}, \rho_{3}\right)$ respectively. The current is in fact determined by the density constraint $(1-f) \rho_{1,4}\left(J_{0}\right)+f \rho_{2,3}\left(J_{0}\right)=\rho$. The variation of density between $\rho_{1}$ and $\rho_{2}$ (or $\rho_{3}$ and $\rho_{4}$ ) between $\mathrm{X}$ and Y stretches corresponds to the 'sub-bands' seen in Fig. (10a). On a macroscopic scale, however, the system has uniform density. Now consider what happens when the mean density is brought closer to $1 / 2$. From Fig. 11, it is evident that the current would tend to increase, and would eventually reach the maximum allowed value $J_{\max }^{Y}$ (which equals 1/4 in the thermodynamic limit, as argued 
below). As the density is increased further, the current remains constant at $J_{\max }^{Y}$, in accordance with the maximum current principle, and the excess density is taken care of by converting some of the $\mathrm{X}$ stretches of density $\rho_{1}$ into ones with $\rho_{4}$ (or vice versa if $\rho>1 / 2$ ). This conversion takes place adjacent to the largest stretch of $\mathrm{Y}$ bonds, leading to two macroscopic regions of different mean densities - one with densities $\left(\rho_{1}, \rho_{2}\right)$ for the $\mathrm{X}$ and $\mathrm{Y}$ stretches, and the other with $\left(\rho_{4}, \rho_{3}\right)$. The position of the principal shock separating these regions is at the location of the largest $\mathrm{Y}$ stretch. In the DASEP, the assumption of uniform density in each stretch is not really true, on account of the finite length of most of the stretches. However, the above argument provides a qualitative picture towards understanding the reason for phase separation in the DASEP.

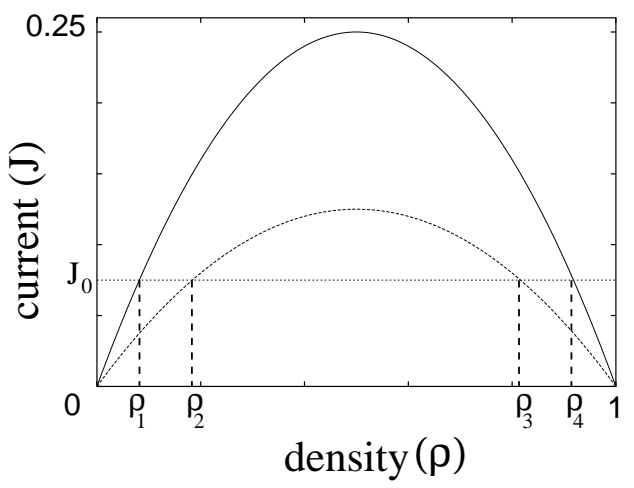

FIG. 11. Origin of phase separation in DASEP. The two parabolas $J=r \rho(1-\rho) ; r=1,1 / 2$, represent the $J-\rho$ plots for the two reference non-disordered systems.

In a certain respect, the reason for the phase separation in the DASEP is similar to that in the single defect bond model studied in [11] — both have local segments in the system where the maximum allowed current is less than that allowed everywhere else. In the single defect bond model, phase separation takes place when the current carried by the rest of the system, with presumed uniform density, is larger than the maximum current allowed through the weak bond. In the DASEP, with an extensive number of weak bonds, the largest stretch of weak bonds acts as the current-limiting segment. The length of this stretch increases as $\ln L$ with system size, and in the thermodynamic limit the maximum allowed current in this stretch is $\frac{1}{4} r$ - equal to the maximal current in a pure system with only weak bonds.

The essential point leading to phase separation is thus the maximum current principle, coupled with localized current-limiting regions in the system. In the DASEP, this limit is determined by long stretches of weak bonds, and similar considerations should apply in related models. Consequently, we would expect a density-segregated phase in disordered versions of models which, in the absence of disorder, display a maximum in the steady-state current as a function of density.

\section{E. The Fully Segregated Model}

It is useful to define a model for which many of the approximations made in the previous subsection are actually exact. To this end, we study a Fully Segregated Model (FSM), which is obtained from the binary random model above by arranging all like bonds together. Thus, in this model, one has two large stretches of $\mathrm{X}$ and $\mathrm{Y}$, of lengths $(1-f) L$ and $f L$ respectively. For the FSM in the thermodynamic limit, the assumption of uniform density within each stretch is justified, as correlations due to the junctions decay with increasing separation, and may be neglected in the bulk [24].

Steady-state MC density profiles for the FSM at three different fillings $\rho \leq 1 / 2$ are shown in Fig. 12 - symmetry under particle-hole exchange implies analogous behaviour for $\rho \geq 1 / 2$ (Appendix A). For low densities $\left(\rho<\rho_{c}^{-}\right)$, the two stretches have uniform bulk densities $\rho_{x}$ and $\rho_{y}$ related to each other by the requirement of equality of the two bulk currents,

$$
\rho_{x}\left(1-\rho_{x}\right)=r \rho_{y}\left(1-\rho_{y}\right)=J_{0},
$$

and the density constraint

$$
(1-f) \rho_{x}+f \rho_{y}=\rho .
$$

These three equations determine $\rho_{x}, \rho_{y}$ and $J_{0}$ uniquely for a given $\rho$. For $f=1 / 2$ we obtain

$$
\begin{aligned}
& \rho_{y}=\frac{4 \rho-1-r \pm \sqrt{(4 \rho-1-r)^{2}+8(1-r) \rho(1-2 \rho)}}{2(1-r)} \\
& \rho_{x}=2 \rho-\rho_{y}, \quad J_{0}=\rho_{x}\left(1-\rho_{x}\right)
\end{aligned}
$$

This is analogous to the macroscopically homogeneous state of the fully random system.

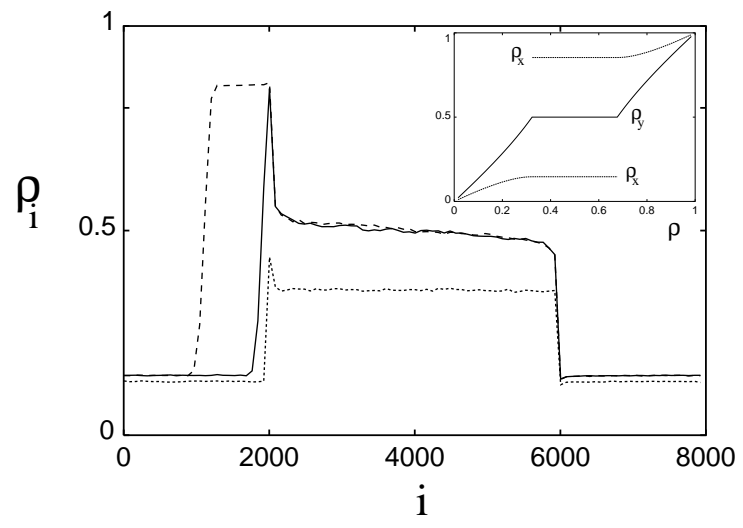

FIG. 12. Representative density profiles of the FSM with $r=f=1 / 2$ at three different fillings: (a) $\rho=0.24$ (dotted) (b) $\rho=\rho_{c}^{-}=0.324$ (solid) and (c) $\rho=0.4$ (dashed). The Y stretch $(r=1 / 2)$ is in the range $i \in[2000,6000]$. The inset shows the variation of the bulk densities in the $\mathrm{X}$ and $\mathrm{Y}$ stretches as a function of the filling $\rho$. 
As the mean density is increased, the density of each stretch increases, until, at a critical density $\rho=\rho_{c}^{-}=$ $\frac{1}{2}-\frac{1}{4} \sqrt{1-r}$ (the corresponding critical density on the higher side is $\rho_{c}^{+}=1-\rho_{c}^{-}$), $\rho_{y}$ equals $1 / 2$ (Fig. 12). At this density, the current equals the maximum possible current in Y, namely, $J_{0}=J_{\max }=r / 4$. As $\rho$ is increased further, $\rho_{y}$ and $J_{0}$ remain constant at $1 / 2$ and $r / 4$ respectively. The density change is adjusted by creating a density inhomogeneity in stretch $\mathrm{X}$. The two densities $\rho_{x}^{\text {high }}$ and $\rho_{x}^{\text {low }}$ are related by $\rho_{x}^{\text {high }}+\rho_{x}^{\text {low }}=1$ so that the currents in the two phases are equal, i.e. $\rho_{x}^{\text {high }}\left(1-\rho_{x}^{\text {high }}\right)=\rho_{x}^{\text {low }}\left(1-\rho_{x}^{\text {low }}\right)=r / 4$. This implies $\rho_{x}^{\text {high,low }}=(1 \pm \sqrt{1-r}) / 2$ (see inset in Fig. 12). The fraction of these phases can be determined from a lever rule and are given by $f^{\text {high,low }}=\left|\rho-\rho_{c}^{\mp}\right| /\left(\rho_{x}^{\text {high }}-\rho_{x}^{\text {low }}\right)$. This locking of the density in the $\mathrm{Y}$ stretch at $\rho_{y}=1 / 2$ is a direct consequence of the maximum current principle introduced above: any change of $\rho_{y}$ from $1 / 2$ would reduce the current in $\mathrm{Y}$, and hence in the full system. All the arguments above can be applied for $\rho>1 / 2$ because of particle-hole symmetry. Thus for $\rho_{c}^{-}<\rho<\rho_{c}^{+}$the state of the segregated model is analogous to the phase separated regime $\mathrm{B}$ of the random model. The size of regime $\mathrm{B}$ in the FSM is given by $2 \Delta=\rho_{c}^{+}-\rho_{c}^{-}=\sqrt{1-r} / 2$. It closely approximates the size of the $\mathrm{B}$ regime in the DASEP (Fig. 9).

\section{F. Phase-coexistence curve}

For the FSM, as $r$ is varied we obtain different $J_{0}$ vs $\rho$ curves. The phase-coexistence curve in the currentdensity plane in the parametric form

$$
J_{c}=\frac{r}{4}, \quad \rho_{c}=\frac{1}{2} \pm \frac{1}{4} \sqrt{1-r}
$$

which is the parabola $J_{c}=1 / 4-\left(1-2 \rho_{c}\right)^{2}$ in Fig. 13.

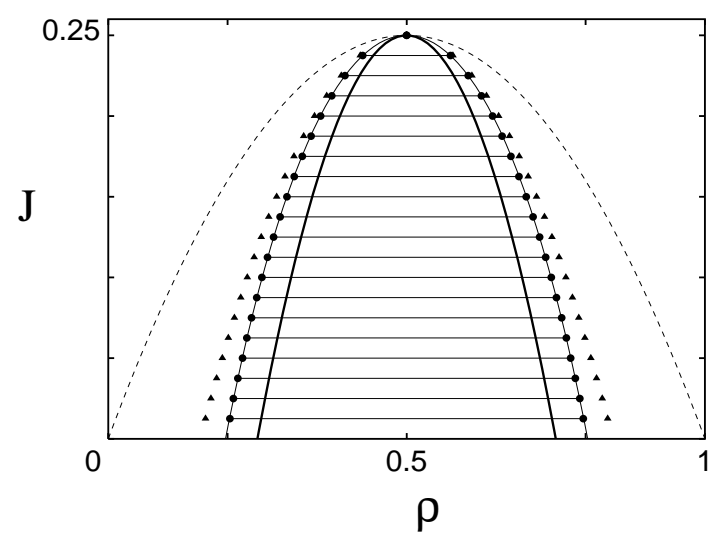

FIG. 13. The phase coexistence curve for the FSM and the DASEP for $f=1 / 2$. The solid parabola is the coexistence curve of the FSM. The circles and the triangles are respectively the MC and mean-field phase-coexistence curves for the DASEP. The dashed parabola $J=\rho(1-\rho)$ demarcates the allowed region for the DASEP.
The difference between the phase boundaries of the DASEP and FSM Fig. 13 comes from the fact that the interspersed weak-bond stretches in the DASEP have finite lengths, and the density in these small stretches is not quite equal to $1 / 2$. Close to the phase separation, we anticipate the mean density in a Y-stretch of length $l$ in the DASEP to be of the form $\rho_{Y}(l)=1 / 2 \pm A(r) / l^{\alpha}(r)$, with $\alpha(r)>0$. Using the distribution of the $Y$ stretches, namely $P_{Y}(l)=2^{-l}$, we obtain the $r$ dependence of the critical density

$$
\rho_{c}^{ \pm}=\frac{1}{4}+\rho_{X} \pm A(r) \sum_{l} P_{Y}(l) \rho_{Y}(l)
$$

where $\rho_{X}(r<<1) \sim r$ is the density in the $\mathrm{X}$ stretches near the phase transition. Comparing with the phase diagram for the FSM in Fig. 13, the correction term $A(r)$ seems to be positive for all $r$. Further, let us suppose that the current in the FSM is a lower bound to that in the DASEP, as suggested by Fig. 13. Then the coexistence curve for the random system must be quadratic near the critical point $\left(J^{0}=1 / 4, \rho^{0}=1 / 2\right)$ - being bounded by two quadratics, namely, the $J-\rho$ curve for the pure system $J=\rho(1-\rho)$, and the coexistence curve of the FSM $J=1 / 4-(1-2 \rho)^{2}$.

\section{G. Correlations in the steady state}

Figure 14 shows the Monte Carlo results for the siteaveraged density-density and height-height correlation functions $\bar{G}(\Delta r)$ and $\overline{\Gamma^{2}}(r)$ in both the homogeneous and density-segregated regimes of the DASEP. $\bar{G}(\Delta r)$ is seen to decay rapidly over a few lattice spacings, accounting for the success of the mean field approximation. It is found that $\overline{\Gamma^{2}}(r)$ grows as $r$ - implying a roughness exponent $\alpha=1 / 2$.

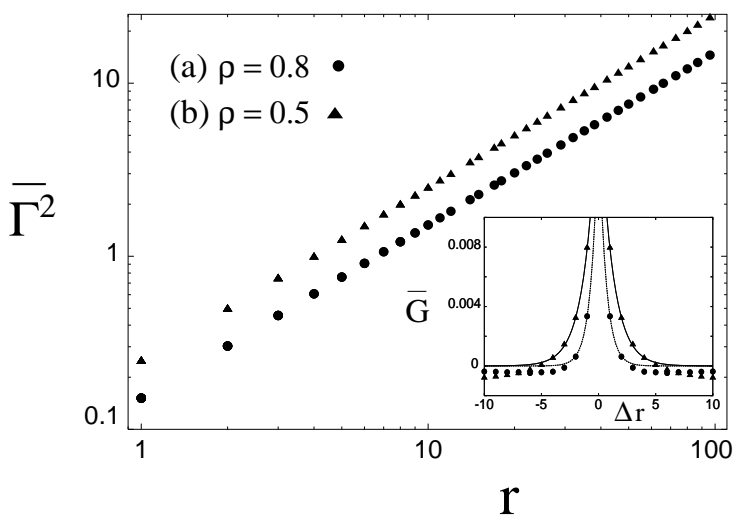

FIG. 14. Height-height correlation function $\overline{\Gamma^{2}}(r)$ for DASEP: (a) in Homogeneous (circles), and (b) Segregated-Density (triangles) regimes. Inset shows the site averaged density-density correlation function $\bar{G}(\Delta r)$ defined in (15). The small negative values at large $|\Delta r|$ arise due to the finite size of the system. 


\section{DASEP WITH BACKBENDS}

As discussed at the beginning of Section [II], the introduction of randomness in the easy direction of individual bonds alters the properties of one-dimensional disordered exclusion process in a crucial way. We study this in this section.

The model is defined as follows: Assign quenched arrows (pointing either right or left) independently to each bond of a periodic chain, with probability $f<\frac{1}{2}$ for left arrows, and $1-f$ for right arrows. An arrow defines the easy direction of hopping on each bond: a particlehole exchange across a bond occurs with rate $w(1+g)$ if the particle moves along the direction of the arrow, and $w(1-g)$ if it moves opposite to the arrow. Since $f<\frac{1}{2}$, there is an overall tendency for particles to circulate rightward, and the question is whether there is a nonzero current even in the thermodynamic limit.

The model represents a system of hard-core particles in a random potential with a downward tilt. A conglomeration of left pointing arrows constitutes a backbend, where the potential climbs up before going down again. Within mean-field theory it is possible to obtain an upper bound $J_{l}$ on the current that can be carried by mutually excluding particles through a backbend of length $l$ [5]. To this end, consider biased diffusion of hard core particles in the segment $[0, l]$ of a 1-d lattice, with the 'optimal' boundary conditions $\rho(0)=1$ and $\rho(l)=0$; these boundary conditions force the largest possible current through the segment, opposite to the bias. The master equation that describes transport is invariant under interchange of particles and holes and simultaneous relabelling of sites in reverse order, i.e. $n_{j} \rightarrow \bar{n}_{l-j}$ The boundary conditions respect this symmetry, implying that the steady-state density $\rho(j)$ at site $j$ satisfies $\rho(j)=1-\rho(l-j)$. Thus in the steady state the number of particles in the backbend is $l / 2$ irrespective of the strength of the bias $g$. The principal effect of increasing $g$ is to sharpen the region which marks the transition from the particle-rich half of the backbend to the hole-rich half. The steady-state profile approaches a step function centered at $j=l / 2$ as $g \rightarrow 1$.

The current in the steady state is the number of particles crossing site $l$ in unit time. Results of a Monte Carlo study [5] are consistent with the large-l asymptotic behaviour

$$
J_{l} \sim \exp \left(-\frac{1}{2} l / \Lambda(g)\right) .
$$

where $\Lambda(g)$ is a bias-induced length given by $\Lambda^{-1}(g)=$ $\ln \{(1+g) /(1-g)\}$. This can be seen by writing the current within a mean field approximation as $J=W(1+$ g) $\rho_{j}\left(1-\rho_{j+1}\right)-W(1-g) \rho_{j+1}\left(1-\rho_{j}\right)$, and finding the value of $J$ for which the boundary conditions $\rho_{0}=1$, $\rho_{l}=0$ hold. For $l>>(g)>>1$, this leads to $J \approx 2 g e^{-l g}[5]$, in agreement with (37) when $g$ is small.

The origin of the factor $\frac{1}{2}$ in the exponent in (37) has been discussed in [5], and we recount the argument in brief. The transport of a single particle through the backbend involves two (approximately) causally independent steps which occur in parallel: (i) the topmost particle (located at site $k \approx l / 2$ in large fields) has to be activated a distance $l / 2$, which requires an activation time $\tau_{1 / 2} \sim \exp \left(\frac{1}{2} l / \Lambda(g)\right)$ and, (ii) the consequent hole that remains in the steady-state distribution moves to the bottom and is filled up, by moving each of $l / 2$ particles up through a lattice spacing. The time required is $\tau_{1 / 2}$ again. The current $J$ is thus proportional to $\tau_{1 / 2}^{-1}$, and consequently follows 37 ).

Since, for fixed $g$, the largest current that can flow through a long backbend $(l>>\Lambda(g))$ is exponentially small in its length, the largest current through the 1-d lattice of length $L$ is determined by the length $l^{*}(L)$ of the largest backbend encountered. Since the probability of occurrences of $l$ consecutive left-pointing arrows on bonds is proportional to $f^{l}$, we may estimate $l^{*}$ from $L f^{l^{*}}=C$ where $C$ is a constant of order unity. Substituting in (37), we find that the current falls with increasing lattice size as

$$
J(L) \sim L^{-\frac{1}{2} \theta}
$$

with $\theta^{-1}=\Lambda(g) \ln f$. Thus the current is expected to decay as a power law in $L$, with a bias-dependent power, and to vanish in the thermodynamic limit. Figure 15 shows the result of Monte Carlo simulations.

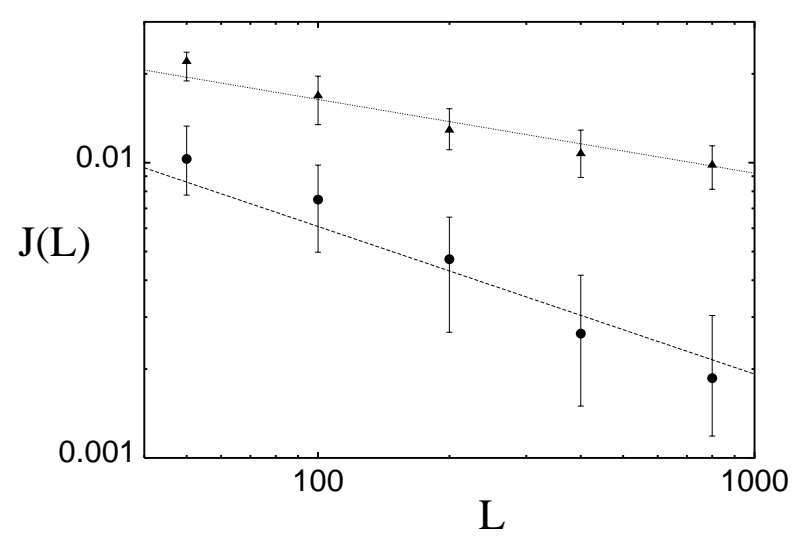

FIG. 15. Size dependence of the steady-state current in the backbend model (DASEP with some reversed bonds) for two sets of parameters: (a) $g=0.33, f=0.25$ (triangles) and (b) $r=0.54, f=0.3$ (circles). Each point represents an average over 40 realizations of disorder in (a) and 100 realizations in (b). The straight lines have slopes of $-\theta / 2$ with $\theta=0.5$ and 1.0 respectively as predicted by $(38)$.

As with the milder sort of disorder discussed in Section III, the state is strongly inhomogeneous and shows macroscopic regions of high and low density. Figure $\mathbb{1} \mathrm{c}$ shows the time-averaged density profile for a typical configuration of bonds. There is a large shock around the rate-limiting backbend, which separates the two regions. 
For fixed lattice size $L$ with an associated longest backbend $l^{*}(L)$, the current is a nonmonotonic function of $g$. This can be seen as follows. If $g$ is low enough that $\Lambda(g)>>l^{*}(L)$, linear response theory would imply that current $J$ grows linearly with $g$. On the other hand, if $g$ is large enough that $\Lambda(g)<<l^{*}(L)$, the current falls with increasing $g$ according to (37). In between, $J$ achieves a local maximum when $\Lambda\left(g=g_{\max }\right) \simeq l^{*}(L)$, which implies $g_{\max } \sim 1 / \ln L$.

The argument given above implies that the current carried by a system of hard-core particles through the randomly backbending lattice vanishes in the thermodynamic limit, no matter how small the bias $g$. This is in contrast to the behaviour of noninteracting particles in the same random environment, where the drift velocity vanishes only if the bias is strong enough [25,60. The difference can be traced to the possibility, in the noninteracting case, of compensation by a large build-up of density at the bottom of a backbend, which then succeeds in driving a finite current over the backbend. This option does not exist once repulsive hard-core interactions come into play, and the current vanishes in the thermodynamic limit.

\section{CONTINUUM DESCRIPTION}

It is interesting to ask whether the behaviours found above in the disordered lattice gases can be reproduced using a continuum description of the problem. Though we have not pursued this question to its logical end, we discuss in this section some general constraints that a continuum description should satisfy.

The steady-state of the disordered system of interacting particles is described by a spatially varying timeaveraged density profile $\rho_{0}(r) \equiv\langle n(r)\rangle$. The time evolutions of fluctuations around the mean density profile are governed by the continuity equation

$$
\frac{\partial}{\partial t} \rho(r, t)+\nabla \cdot \vec{J}(\rho, r, t)=0
$$

The coarse-grained current $\vec{J}$ may be phenomenologically divided into three parts

$$
\vec{J}(r, t)=\vec{J}_{s y s}(\rho(r, t), r)+\vec{J}_{d i f f}+\vec{\epsilon}(r, t) .
$$

A local hydrodynamic assumption has been made in writing the systematic part of the current, $\vec{J}_{\text {sys }}$ as a function of the local density, $\rho(r, t)$. The explicit $r$ dependence of $\vec{J}_{\text {sys }}$ comes from the breaking of translational invariance by quenched disorder, and its exact form of $\vec{J}_{s y s}$ depends on the microscopic dynamics of the model. The diffusive current, $\vec{J}_{\text {diff }}=D(r) \cdot \nabla\left[\rho(r, t)-\rho_{0}(r)\right]$, involves a quenched diffusion tensor, $D(r)$. The noise, $\vec{\epsilon}(r, t)$, is to mimic, on a mesoscopic scale, the stochastic nature of the evolution. It is usually assumed to be Gaussian distributed and $\delta$-correlated in space and time with vanishing spatial and temporal averages.

To obtain the time evolution of the density fluctuations $\tilde{\rho} \equiv \rho(r, t)-\rho_{0}(r)$, we expand $\vec{J}_{\text {sys }}$ in powers of $\tilde{\rho}$ as $\vec{J}_{\text {sys }}\left(\rho_{0}(r), r\right)+\vec{c}(r) \tilde{\rho}+\vec{\lambda}(r) \tilde{\rho}^{2} \cdots$ and put it in 39 . This results in

$$
\partial_{t} \tilde{\rho}=\nabla \cdot\left[D(r) \cdot \nabla \tilde{\rho}-\vec{c}(r) \tilde{\rho}-\vec{\lambda}(r) \tilde{\rho}^{2} \cdots-\vec{\epsilon}(r, t)\right]
$$

In one spatial dimension, the above reduces to (with $r$ replaced with $x$ ) the form

$$
\partial_{t} \tilde{\rho}=\partial_{x}\left[D(x) \partial_{x} \tilde{\rho}-c(x) \tilde{\rho}-\lambda(x) \tilde{\rho}^{2} \cdots-\eta(x, t)\right]
$$

which was considered in [2]. In steady state, the timeaveraged current must be independent of $x$. As both $J_{\text {diff }}$ and $\epsilon(x, t)$ vanish under time averaging, the constraint to be satisfied is

$$
\partial_{x}\left\langle J_{s y s}(x)\right\rangle=0
$$

which is important to account for, as the coefficients in $J_{\text {sys }}$ are explicitly space-dependent, i.e., $\partial_{x}\left[\lambda(x)\left\langle\tilde{\rho}^{2}\right\rangle+\ldots\right]$ must vanish.

In their attempt to study a continuum model which describes the DASEP, Becker and Janssen (BJ) [8] write the current $J^{\prime}$ in powers of $\phi(x, t) \equiv \rho(x, t)-\rho$, the density fluctuation away from the overall particle density $\rho$ in the system. In one dimension, the form quoted [3] for the DASEP is

$$
J^{\prime}=(1-2 \rho) \phi(x, t)-\phi^{2}(x, t)+\eta(x)
$$

where $\eta(x)$ is an additive quenched noise term. Since the time-averaged density $\rho_{0}(x)$ varies in space, it is evident that $\phi$ has a nonzero expectation value $\langle\phi(x, t)\rangle=$ $\rho_{0}(x)-\rho$. As we have seen in Section III, $\rho_{0}(x)$, and thus $\langle\phi(x, t)\rangle$, can show strong variations, especially in the density-segregated regime. Spatial constancy of the average current demands that the time average of the right hand side of (44) must satisfy

$$
\partial_{x}\left[(1-2 \rho)\langle\phi(x, t)\rangle-\left\langle\phi^{2}(x, t)\right\rangle+\eta(x)\right]=0 .
$$

Even when this condition is satisfied, it is not entirely clear that this continuum theory actually represents the DASEP in one dimension. BJ argue that if $\partial J / \partial \rho \neq 0$, then disorder is irrelevant on the large scale, a conclusion that is supported by [2, 26. However, their conclusion, that the condition $\partial J / \partial \rho=0$ holds only if $\rho=\frac{1}{2}$, does not seem to be correct, at least for the DASEP in one dimension; we have seen in Section IV, there is an entire range of densities $\left(\frac{1}{2}-\Delta \leq \rho \leq \frac{1}{2}+\Delta\right)$ where $\partial J / \partial \rho$ vanishes, associated with segregation of density on a macroscopic scale. 


\section{EQUIVALENT INTERFACE MODELS IN ONE DIMENSION}

In 1- $d$, both the DASEP (with or without backbends) and the GDDP - for which the maximum occupancy per site is 1 - are equivalent to stochastic growth models of a $1-d$ interface moving in a $2-d$ medium. Corresponding to each particle-hole configuration $\left\{n_{i}\right\}$ is assigned an interface profile $\left\{H_{i}\right\}$ through $H_{i}=\sum_{j \leq i}\left(1-2 n_{i}\right)[12$. Pictorially this means that each particle corresponds to a $-45^{\circ}$ downward line segment, while a hole corresponds to an upward one (Fig. 16). Thus, clusters of particles and holes translate to $\pm 45^{\circ}$ slope segments, and the interface has a mean slope which vanishes when the particle density is $1 / 2$. Away from half-filling, periodic boundary condition for the lattice becomes a helical boundary condition for the interface. Junctions between adjacent particle and hole clusters correspond to corners in the interface profile.

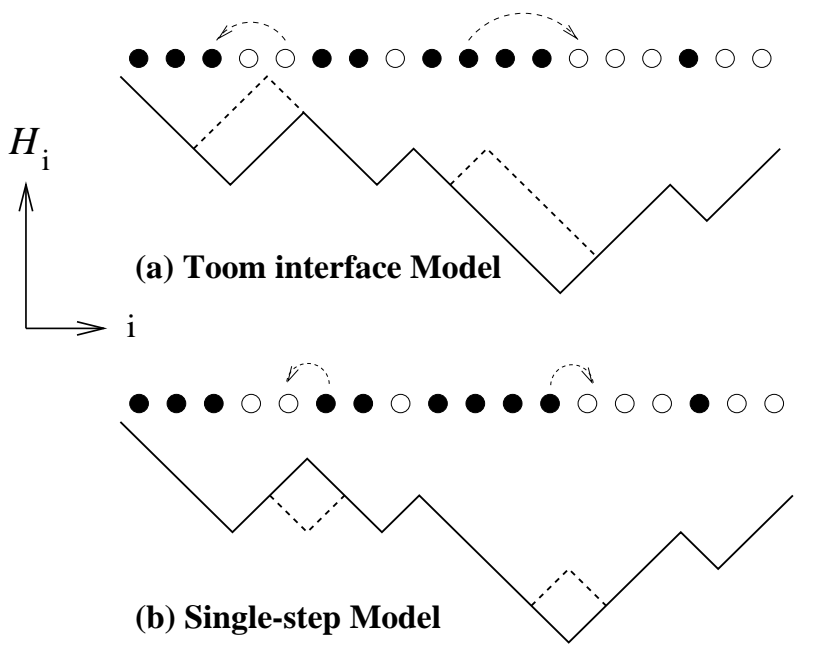

FIG. 16. Mapping between driven particle systems in $d=1$ and growing interfaces. (a) Toom Interface dynamics corresponding to the GDPP, (b) Single-step model corresponding to the DASEP.

Evolution of the interface is dictated by the dynamics of the corresponding particle system. The GDDP corresponds to the slice-wise motion of segments of a Toom interface in the low noise limit [20], while the DASEP corresponds to the corner-flip 'single step' growth model 12 (Fig. 16). In both cases particle movement to the right (or hole move to left in GDDP) corresponds to local forward growth (deposition) of the interface while a leftward particle move (e.g. in DASEP with backbends) corresponds to local backward growth (evaporation). The quenched jump rates for the particle moves implies columnar disordered growth-rates for the interface: the normal growth rate of the interface at a fixed $i$ is the same irrespective of the location of the interface at successive times. In the long time limit, the mean local forward speed of the interface is the same at all points along the interface - being proportional to the spatially constant steady-state current of the corresponding particle model.

We now discuss the various qualitatively different regimes which arise in the interface growth models. Figure 17 shows time averaged steady-state interface profiles $\left\langle H_{i}\right\rangle$ corresponding to the three regimes of driven particle systems illustrated in Fig. 1 in Section If In all the three cases we started from an initially uniform profile - corresponding to a random distribution of particles on the lattice.
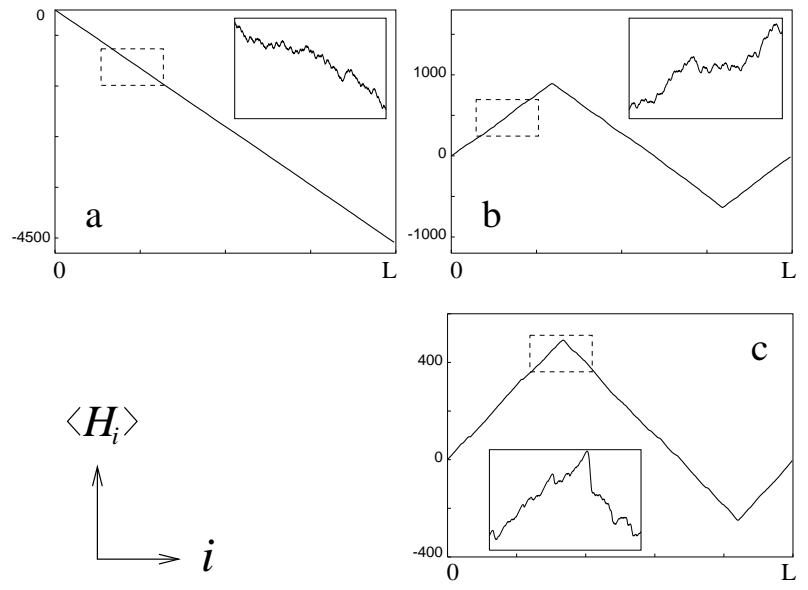

FIG. 17. Interface morphology in the three regimes: (a) Uniformly moving interface with a uniform slope, (b) interface with large segments of different slopes moving with a non-vanishing speed, and (c) interface with large segments of different slopes moving with a speed which vanishes in the thermodynamic limit. The insets show blow-ups of regions enclosed in the dashed boxes.

Figure 17a is an interface with a non-zero mean tilt, which has net forward growth rate at all points. The interface has a uniform slope on a macroscopic scale and moves with a finite non-zero speed preserving its mean tilt. This case corresponds to the Homogeneous regime depicted in Fig. 17a. On a microscopic scale the interface has frozen-in roughness (Fig. 17a, inset) corresponding to the microscopic shocks in the steady-state density profile of Fig. 11a.

If the mean tilt vanishes, but the interface still has a net forward growth rate at all points, then the initial uniform profile at $t=0$ coarsens into large segments of different mean slopes at long times (Fig. 17b). These segments have frozen roughness on microscopic scales, similar to the non-zero tilt case (Fig. 17b, inset). The interface moves with a finite speed preserving its mean shape and mean vanishing tilt. This corresponds to the Segregated-Density regime of Fig. 1 b.

In addition to the frozen roughness on the microscopic scales, we can define the kinetic roughness as the equaltime mean-square height fluctuations around the steadystate profile. We consider the zero-mean height variables 
$h_{i}(t) \equiv H_{i}(t)-\left\langle H_{i}\right\rangle$ defined in (17), and define the roughness exponent $\alpha$ through $\overline{\left\langle\left(h_{i+r}-h_{i}\right)^{2}\right\rangle} \sim r^{2 \alpha}$. As discussed in Subsections IID, E and IIF, the roughness exponent $\alpha=1 / 2$, in both the cases above.

In Fig. 17c, which corresponds to the VanishingCurrent regime of Fig. 1 $\mathrm{c}$, the profile resembles that corresponding to the Segregated-Density regime in that it has large segments of different mean slopes. However, in this case the interface is stationary in the thermodynamic limit - reflecting the vanishing of the steady-state current in the particle system. In interface language, this situation can be visualized as a case of interface growth where there are local stretches of the interface having a net backward growth (evaporation) rate. Though, on the average, there are more forward-growth regions than backward-growth ones, in the limit of large system size, arbitrarily long evaporation stretches effectively pin the interface.

Turning now to continuum description of the dynamics of these interfaces, at least in the cases where the mean speed of growth is non-zero, the sum in the definition of the height variable is replaced by an integral of the coarse-grained particle density, $H(x, t)=\int^{x}[1-$ $\left.2 \rho\left(x^{\prime}, t\right)\right] d x^{\prime}$. The growth equation for $H(x, t)$ is governed by (spatially) integrated one dimensional version of the continuity equation (39): $\partial_{t} H(x, t)=J\left(\partial_{x} H, x, t\right)$. The fluctuations $h(x, t) \equiv H(x, t)-H_{0}(x)$ in $H$ around the steady-state profile $H_{0}(x) \equiv\langle H(x, t)\rangle$, are governed by

$$
\partial_{t} h=D(x) \partial_{x x} h-c(x) \partial_{x} h+\frac{1}{2} \lambda(x) \partial_{x} h^{2} \cdots+2 \eta(x, t)
$$

obtained by integrating (42). The absence of any additive quenched spatial noise term in (46) is due to the spatial constancy of the growth speed of the interface dictated by the same constraint on the steady-state current. In this respect (46) differs from the model discussed in 27] where such a term arises naturally due to absence of any such constraints. As can be readily verified by power counting, an additive quenched columnar term is highly relevant in the RG sense and leads to much rougher interfaces than the $\alpha=1 / 2$ interfaces described by (46).

\section{CONCLUSION}

In this paper, we have studied the stationary currentcarrying states of driven lattice gas models with quenched disordered hopping rates. The principal results are of two types - first the exact determination of the steady states for a class of disordered models, and second the demarcation of distinct regimes of behaviour on macroscopic length scales, as a result of disorder. In this section, we briefly review these results, and discuss some related open problems.

The steady states of a family of disordered models the disordered drop-push process (DDPP), and related models - have been found in all dimensions by an application of the condition of pairwise balance. The result is a product measure state, with site-dependent weights, reflecting the microscopic disorder in the model. The current has been computed as well. The system is characterized by a strictly uniform current density, and a coarsegained particle density that is approximately uniform. On a macroscopic scale, the state is homogeneous.

Disorder can lead to macroscopically non-homogeneous states, as in the 1- $d$ disordered asymmetric simple exclusion process (DASEP). Our numerical and mean-field results show that a macroscopically density-segregated state occurs in the DASEP model with no backbends, for densities in a finite range around half-filling. The origin of density separation is traceable to the existence of a largest current that can be carried by a stretch of weak bonds. This low current can be sustained in the rest of the lattice only by separating the density into distinct large and small values in macroscopic regions of the lattice.

Backbends introduce a third type of possible behaviour in one dimension. Like the stretch of weak forward bonds in the density-segregated regime, a backbend rate-limits the current, leading to density segregation. However, there is an important difference: the longer the stretch of weak forward bonds, the closer is the current to a finite asymptotic value; by contrast, the longer the stretch of reverse-biased bonds, the smaller the current - it decreases exponentially fast with backbend length. Since the probability of occurrence of a backbend decreases exponentially with its length, the result is a current that decreases as a bias-dependent power of the overall size of the lattice.

The crucial physical point which underlies the behaviour in each of these regimes is the requirement that the steady-state current be constant at all points in the system. Continuum field-theoretical approaches must ensure that this local constraint is respected; while this is automatically assured for translationally invariant systems, it may need special care to guarantee in disordered systems.

It would be of interest to generalize our results to higher dimensions and also to include interactions between particles at different sites. A few scattered results along these lines are available.

(i) For the drop-push class of problems, we have seen in Section II that the exact steady state even in higher dimensions is characterized by inhomogeneous product measure. On large scales, this leads to a homogeneous state.

(ii) The transport of particles with hard-core interactions through the infinite cluster of a randomly diluted lattice above the percolation concentration has been studied [5.65. In a certain regime of dilution, backbends act as local traps, but unlike the one-dimensional case considered in Section IV, there exist infinitely long paths on which the length of every backbend is less than a fixed value 
[28.5.6]. The sub-network of such paths is expected to carry a current which then remains finite in the thermodynamic limit. There is thus no vanishing-current regime in this system.

(iii) With attractive interactions between particles, the driven lattice gas system with nearest-neighbour hopping is known to undergo phase separation below a certain temperature [29,3]. A numerical simulation showed that the addition of a low concentration of blocked sites did not alter the critical behaviour of this system [7].

More systematic studies of higher-dimensional systems are called for. In particular, it would be interesting to know whether disorder-induced large-scale inhomogeneities, akin to the phase separation found in one dimension, persist in higher dimensions as well.

Acknowledgements: We thank R. E. Amritkar, D. Dhar, S. Krishnamurthy, R. Lahiri, G. I. Menon and N. Trivedi for useful discussions.

\section{APPENDIX A: SYMMETRIES OF THE CURRENT IN THE DASEP}

In this appendix we discuss two types of symmetry transformations in the DASEP which leave the steadystate current invariant. (i) The first, which involves flipping the directions of all jumps and interchanging all particles and holes, can be proved exactly. Under these operations, the steady-state weights of particle configurations and the magnitude of the current are shown to remain invariant. (ii) The second type of symmetry is restricted to 1-d systems in which only one way jumps are allowed on each bond. The symmetry transformation consists of flipping the direction of jump on each bond. In this case, we have convincing numerical evidence that the current is invariant, though there appears to be no simple relationship between steady-state weights of various particle configurations. We have no general proof of this result.

(i) Easy direction flip and particle-hole interchange: Consider the DASEP, with quenched disordered, unequal forward and backward hopping rates on each bond. Below we explicitly deal with the one-dimensional case, but the results are easily generalizable to higher dimensions. Let $R$ denote a particular realization of disorder and $\bar{R}$ the realization obtained from $R$ by flipping the easy direction of jumps on all the bonds: If $R$ denotes the set of transition rates $\left\{W^{i j}\right\}$, then $\bar{R}$ corresponds to the set $\left\{\bar{W}^{i j}=W^{j i}\right\}$. Also, let us denote by $\mathcal{C}$ and $\overline{\mathcal{C}}$ two particle configurations related to each other by particle-hole interchange. Clearly, if $\mathcal{C}$ is an allowed configuration of the system at filling $\rho$, then $\overline{\mathcal{C}}$ corresponds to a filling $1-\rho$, and thus there is a one-to-one correspondence between the configurations at the two fillings. Now, let $\mathcal{C}^{i j}$ be the configuration obtained from $\mathcal{C}$ by exchanging the occupation numbers at the sites $i$ and $j$. It is easy to see that the two transition rates, $W_{\rho}\left(\mathcal{C} \rightarrow \mathcal{C}^{i j}\right)$ in realization $R$, and $\bar{W}_{1-\rho}\left(\overline{\mathcal{C}} \rightarrow \overline{\mathcal{C}^{i j}}\right)$ in realization $\bar{R}$ are equal
(Fig. 18), i.e. the transition matrices $\mathcal{W}_{\rho}$ and $\overline{\mathcal{W}}_{1-\rho}$ in the two realizations have identical entries.

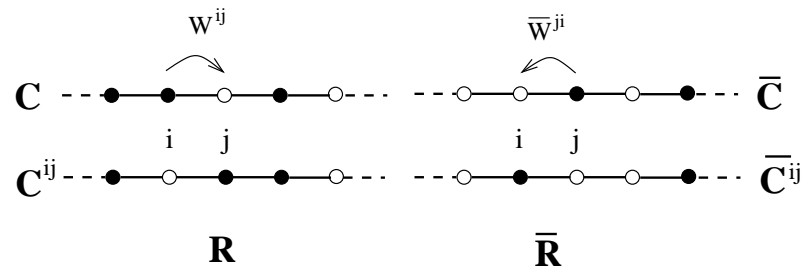

FIG. 18. Invariance of the current under $R \rightarrow \bar{R}$ and $\rho \rightarrow 1-\rho$ for the DASEP in 1- $d$. Jump directions on every bond are reversed from $R$ to $\bar{R}$. Configurations $\mathcal{C}, \overline{\mathcal{C}}$ are related by particle-hole interchange. So are $\mathcal{C}^{i j}$ and $\overline{\mathcal{C}^{i j}}$.

Identification of the two $\mathcal{W}$-matrices implies that the invariant measures $P_{\rho}$ and $\bar{P}_{1-\rho}$ satisfy

$$
P_{\rho}(\mathcal{C})=\bar{P}_{1-\rho}(\overline{\mathcal{C}}) .
$$

Using this, together with the identity $n_{i}(\mathcal{C})=1-n_{i}(\overline{\mathcal{C}})$, we can relate $n$-point correlations at fillings $\rho$ and $1-\rho$. In particular, the site densities at the two fillings are related as

$$
\left\langle n_{i}\right\rangle_{\rho, R}=1-\left\langle n_{i}\right\rangle_{1-\rho, \bar{R}}
$$

Further, the steady-state currents in $R$ and $\bar{R}$ at the two fillings are equal in magnitude:

$$
\begin{aligned}
J_{0}(\rho, R) & \equiv W^{i j}\left\langle n_{i}\left(1-n_{j}\right)\right\rangle_{\rho, R}-W^{j i}\left\langle n_{j}\left(1-n_{i}\right)\right\rangle_{\rho, R} \\
& =\bar{W}^{j i}\left\langle n_{j}\left(1-n_{i}\right)\right\rangle_{1-\rho, \bar{R}}-\bar{W}^{i j}\left\langle n_{i}\left(1-n_{j}\right)\right\rangle_{1-\rho, \bar{R}} \\
& =-J_{0}(1-\rho, \bar{R}) .
\end{aligned}
$$

The negative sign in the last step reflects the fact that the direction of the current is opposite in the two systems.

In the case of the FSM (subsection IIIE) the realizations $R$ and $\bar{R}$ are identical (apart from a reflection). Hence in this case the above arguments imply that the magnitudes of the currents at the two fillings $\rho$ and $1-\rho$ are equal. A similar result holds for the single defect bond case studied by Janowsky and Lebowitz [11].

For the DASEP, with or without backbends, (A3) has the corollary that the disorder averaged currents at the two fillings $\rho$ and $1-\rho$ are equal.

(ii) Easy direction flip only (for 1-d, forward hopping): Above, we found that the current is invariant when the filling is changed from $\rho$ to $1-\rho$, provided that the disorder realization is changed from $R$ to $\bar{R}$. Here we observe (based on numerical evidence) that in 1- $d$, with only forward hopping, the result is also true for realization $R$ on its own, i.e.

$$
J_{0}(\rho, R)=J_{0}(1-\rho, R) .
$$

In view of (A3) this is equivalent to

$$
J_{0}(\rho, R)=-J_{0}(\rho, \bar{R}) .
$$


The claim is easily verified for the single particle (or single hole) case using an explicit form for the current: $J_{0}=\left(\sum_{i} \alpha_{i, i+1}^{-1}\right)^{-1}$ 25. Also, (A5) is true at $\rho=1 / 2$ since (A4) is an identity at this filling.

We do not have a proof for (A4) or (A5) in the general case, but they seem to be borne out numerically. For instance, we studied the validity of (A5) for a system of size $L=6$ with $N=2$ particles. We took $R$ to be the set $\left\{W^{i, i+1}, i=1, \cdots, 6\right\}=\left\{\frac{1}{2}, \frac{1}{2}, 1,1, \frac{1}{2}, 1\right\}$. The invariance of the current is verified up to 1 part in $10^{8}$. We also studied the steady-state probabilities of all the ${ }^{6} C_{2}$ particle configurations for each of $R$ and $\bar{R}$, both by Monte Carlo and by Lanczos iteration of the stochastic evolution operator. There seems to be no straightforward correspondence between the two sets of steady-state weights. This suggests that there should be a proof of the invariance of the current which does not rely on identifying the weights of individual configurations.

[1] D. S. Fisher in Nonlinearity in Condensed Matter edited by A. R. Bishop, D. K. Campbell, P. Kumar and S. E. Trullinger (Springer-Verlag, Berlin, 1987).

[2] A brief account of some of the results in this paper has appeared earlier in G. Tripathy and M. Barma, Phys. Rev. Lett. 78, 3039 (1997).

[3] B. Schmittman and R. K. P. Zia, in Phase Transitions and Critical Phenomena, edited by C. Domb and J. L. Lebowitz (Academic Press, London, 1995), Vol. 17.

[4] J. -P. Bouchaud and A. Georges, Phys. Rep. 195, 127 (1990).

[5] R. Ramaswamy and M. Barma, J. Phys. A 20, 2973 (1987).

[6] M. Barma and R. Ramaswamy in Non-linearity And Breakdown in Soft Condensed Matter, edited by K. K. Bardhan, B. K. Chakrabarti and A. Hansen (Springer, Berlin, 1993), p.309.

[7] K. B. Lauritsen and H. Fogedby, Phys. Rev. E. 47, 1563 (1992).

[8] V. Becker and H. K. Janssen, Europhys. Lett. 19, 13 (1992).

[9] The disorder in our models is quenched in space, in contrast to models in which quenched jump rates are associated with particles, as in M. R. Evans, Europhys. Lett. 36, 13 (1996) and J. Krug and P. A. Ferrari, J. Phys. A
29, L465 (1996).

[10] G. Schütz, R. Ramaswamy and M. Barma, J. Phys. A29, 837 (1996).

[11] S. A. Janowsky and J. L. Lebowitz, Phys. Rev. A 45, 618 (1992); S. A. Janowsky and J. L. Lebowitz, J. Stat. Phys. 77, 35 (1994).

[12] J. Krug and H. Spohn in Solids Far from Equilibrium: Growth, Morphology and Defects, edited by C. Godréche (Cambridge University Press, Cambridge, 1991).

[13] J. Krug and T. Halpin-Healy, J. Phys. (France) I 3, 2179 (1993); T. Halpin-Healy and Y. -C. Zhang, Phys. Rep. 254, 215 (1995).

[14] O. Narayan and D.S. Fisher, Phys. Rev. B 49, 9469 (1994).

[15] This set of numbers can in general may be any set of positive numbers. In the disordered case, they are chosen from a probability distribution. See, for example, subsection II E where explicit results are obtained if these rates are chosen from a binary distribution.

[16] This model of quenched randomness with wells of different depths has been referred to as the 'random trap' model in connection with single particle diffusion. e.g. see J. W. Haus and K. W. Kehr, Phys. Rep. 150, 263 (1987).

[17] N. G. van Kampen, Stochastic Processes in Physics and Chemistry (North Holland, Amsterdam, 1992).

[18] P. -M. Binder, M. Paczuski and M. Barma, Phys. Rev. E 49, 1174 (1994).

[19] Here the angular brackets $\langle\cdot\rangle$ denote time average in the steady state which, in view of the ergodicity of the dynamics, is identical to the expectation value with respect to the invariant measure.

[20] B. Derrida, J. L. Lebowitz, E. R. Speer and H. Spohn, Phys. Rev. Lett. 67, 165 (1991).

[21] T. M. Liggett, Interacting Particle Systems, Springer, New York (1985).

[22] H. Spohn, Large Scale Dynamics of Interacting Particles (Springer, Berlin, 1991).

[23] J. Krug, Phys. Rev. Lett. 67, 1882 (1991).

[24] The density profile approaches the bulk value as a power of the distance from the junctions. See G. Tripathy in Proceedings of the 4th Taipei International Workshop and Symposium on Statistical Physics 1997, edited by C. -K. Hu et. al. (North-Holland, Amsterdam), to appear.

[25] M. Barma and D. Dhar, J. Phys. C 16, 1451 (1983).

[26] G. Tripathy and M. Barma, unpublished.

[27] J. Krug, Phys. Rev. Lett. 75, 1795 (1995).

[28] M. Barma and R. Ramaswamy, J. Phys. A 19, L605 (1986).

[29] S. Katz, J. L. Lebowitz and H. Spohn, J. Stat. Phys. 34, 497 (1984). 